\title{
The extremely rapid rotational braking of the magnetic helium-strong star HD 37776 ^
}

\author{
Z. Mikulášek ${ }^{1,2}$, J. Krtička ${ }^{1}$, G. W. Henry ${ }^{3}$, J. Zverko ${ }^{4}$, J. Žižňovský ${ }^{4}$, D. Bohlender ${ }^{5}$, I. I. Romanyuk ${ }^{6}$, J. Janík ${ }^{1}$, \\ H. Božicic ${ }^{7}$, D. Korčáková ${ }^{8}$, M. Zejda ${ }^{1}$, I. Kh. Iliev ${ }^{9}$, P. Škoda ${ }^{8}$, M. Šlechta ${ }^{8}$, T. Gráf ${ }^{2}$, M. Netolický1 ${ }^{1}$, and M. Ceniga ${ }^{1,8}$ \\ ${ }^{1}$ Institute of Theoretical Physics and Astrophysics, Masaryk University, Kotlářská 2, 61137 Brno, Czech Republic \\ e-mail: mikulas@physics.muni.cz \\ 2 Observatory and Planetarium of J. Palisa, VŠB - Technical University, Ostrava, Czech Republic \\ 3 Center of Excellence in Information Systems, Tennessee State University, Nashville, Tennessee, USA \\ 4 Astronomical Institute, Slovak Academy of Sciences, Tatranská Lomnica, Slovak Republic \\ 5 National Research Council of Canada, Herzberg Institute of Astrophysics, 5071 W. Saanich Rd., Victoria, BC V9E 2E7, Canada \\ 6 Special Astrophysical Observatory of the Russian AS, Nizhnyi Arkhyz, Russia \\ 7 Hvar Observatory, Faculty of Geodesy, Zagreb University, Zagreb, Croatia \\ 8 Astronomical Institute, Academy of Science of CR, Ondřejov, Czech Republic \\ 9 National Astronomical Observatory Rozhen, Bulgarian Academy of Sciences, Bulgaria
}

Received 4 May 2007 / Accepted 8 April 2008

\section{ABSTRACT}

\begin{abstract}
Context. Light and spectrum variations of the magnetic chemically peculiar $(\mathrm{mCP})$ stars are explained by the oblique rigid rotator model with a rotation period usually assumed to be stable on a long time scale. A few exceptions, such as CU Vir or 56 Ari, have been reported as displaying an increase in their rotation period. A possible increase in the period of light and spectrum variations has also been suggested from observations of the helium-strong mCP star HD 37776 (V901 Ori).

Aims. In this paper we attempt to confirm the possible period change of HD 37776 and discuss a possible origin of this change as a consequence of i) duplicity; ii) precession; iii) evolutionary changes; and iv) continuous/discrete/transient angular momentum loss. Methods. We analyse all available observations of the star obtained since 1976. These consist of 1707 photometric measurements obtained in $u v b y(\beta),(U) B V, V, B_{\mathrm{T}} V_{\mathrm{T}}$, and $H_{\mathrm{p}}$, including 550 of our own recent observations obtained in 2006 and 2007,53 spectrophotometric measurements of the He I $\lambda 4026 \AA$ line, 66 equivalent width measurements of He I spectral lines from 23 CFHT spectrograms acquired in 1986, and $69 \mathrm{He}$ I equivalent measurements from spectral lines present in 35 SAO Zeeman spectrograms taken between 1994 and 2002. All of these 1895 individual observations obtained by various techniques were processed simultaneously by means of specially developed robust codes.

Results. We confirm the previously suspected gradual increase in the 1.5387 period of HD 37776 and find that it has lengthened by a remarkable $17.7 \pm 0.7 \mathrm{~s}$ over the past 31 years. We also note that a decrease in the rate of the period change is not excluded by the data. The shapes of light curves in all colours were found to be invariable.

Conclusions. After ruling out light-time effects in a binary star, precession of the rotational axis, and evolutionary changes as possible causes for the period change, we interpret this ongoing period increase as a braking of the star's rotation, at least in its surface layers, due to the momentum loss through events or processes in the extended stellar magnetosphere.
\end{abstract}

Key words. stars: chemically peculiar - stars: early-type - stars: variables: general - stars: individual: HD 37776

\section{Introduction}

The object HD 37776 (HIP 26742, V901 Ori) is a B2 IV star residing in the reflection/emission nebula IC 432 (van den Bergh 1966; Finkbeiner 2003) and classified as an intermediate heliumrich or a helium-strong, chemically peculiar $(\mathrm{CP})$ star by Hunger (1975).

Photometric variability in HD 37776 was first mentioned by Lynds (1959) and then confirmed by Hill (1967) who, on the basis of his $U B$ photometry, determined a period $0 \mathrm{~d} 37968$ and suggested a tentative classification of the star as a pulsating

\footnotetext{
* Based in part on observations obtained at the Canada-France-Hawaii Telescope (CFHT) which is operated by the National Research Council of Canada, the Institut National des Sciences de l'Univers of the Centre National de la Recherche Scientifique of France, and the University of Hawaii.
}

$\beta$ Cep variable. Unfortunately Hill's observations are not available to check, but his results were questioned by Pedersen \& Thomsen (1977) and Pedersen (1979) who obtained 54 highquality uvby observations and a total of 53 spectrophotometric measurements of the He I $\lambda 4026$ line. The latter observations confirmed the first identification of the star as a probable spectrum variable (Nissen 1976). From the He I spectrophotometric data Pedersen (1979) determined a period of $1.5385 \pm 0.003$. Bartolini et al. (1982) confirmed this longer photometric period from their $U B V$ photometry. The star was also observed by HIPPARCOS (ESA 1997): there are 103 "Hipparcos" $H_{\mathrm{p}}$ measurements and 129 Tycho $B_{\mathrm{T}} V_{\mathrm{T}}$ measurements, the latter analogs of Johnson $B$ and $V$ photometry.

The variations of the helium lines and lines of other singlyand doubly-ionized elements in HD 37776 and other heliumstrong stars are usually interpreted in the framework of the 
magnetic oblique rotator model (Stibbs 1950). The derived period of the photometric and spectroscopic variations is then assumed to be the rotation period of the star.

In the case of HD 37776, the requisite magnetic field was first discovered by Borra \& Landstreet (1979). Additional field measurements by Thompson \& Landstreet (1985) show that the star has an extraordinary double-wave magnetic curve with a period of 1.53869 and they argue that the star has a dominantly quadrupolar field geometry. Bohlender $(1988,1994)$ subsequently showed that the longitudinal magnetic field variations could be reproduced with a combination of aligned dipole, quadrupole and octopole components, with the quadrupolar component dominating the field curve. This star also may have the most pronounced photospheric line profile variations observed in the helium-strong stars (Bohlender 1988, 1994).

Shore \& Brown (1990) demonstrate that the CIV and Si IV resonance doublets of HD 37776, assumed to originate in trapped hot magnetospheric plasma, also vary on the above period but they find that the variations have a smaller amplitude and more complicated behaviour than for other members of the helium-strong class, possibly because of the complex magnetic field geometry of the star. Shore (1987) discussed the consequences of the interaction of radiatively driven mass loss with magnetic fields in upper-main-sequence helium-peculiar stars. He found the fields constrain mass loss to occur in narrow cones above the magnetic poles that can explain the observed behaviour of their UV resonance lines.

Adelman (1997) used additional uvby photometric measurements to derive a more precise rotation period for HD 37776 and provided the following ephemeris

$\mathrm{JD}\left(B_{1}^{+}\right)=2445724.669(20)+1.538675(5) \times E$,

where the phase zero is set to the first magnetic maximum $\left(B_{1}^{+}\right)$ after Thompson \& Landstreet (1985). Each colour light curve has a single maximum, which occurs near phase 0.75 when the surface field predicted by Bohlender (1988) is at its maximum.

Using their own method of Doppler-Zeeman mapping Khokhlova et al. (2000) analysed 14 high-dispersion Zeeman spectrograms and revealed a complex spotty distribution of several chemical elements apparently structured after the stellar magnetic field. The maps show that silicon is, as a rule, overabundant in regions where helium is underabundant and vice versa, confirming the unpublished work of Bohlender (1988).

Using the Khokhlova et al. (2000) surface abundance distributions for helium and silicon, Krtička et al. (2007) calculated theoretical uvby photometric maps and show that the silicon-rich abundance patches result in optically brighter regions than regions with an overabundance of helium. Their model uvby light curves combining the effect of the inhomogeneous surface abundance of both elements fit the observed light curves very well.

Based on a new analysis of the photometric data discussed briefly above (Pedersen \& Thomsen 1977; Pedersen 1979; Adelman \& Pyper 1985; ESA 1997; Adelman 1997), Mikulášek et al. (2007b) have suggested that the photometric period of HD 37776 has increased. In this paper, we present a more detailed analysis and interpretation of a more comprehensive collection of archival and new photometric and spectroscopic observations of HD 37776 obtained at ten epochs distributed quite evenly over more than three decades. We confirm the previously suspected gradual increase of the 1.5387 period of HD 37776 and find that it has lengthened by a remarkable $17.7 \pm 0.7 \mathrm{~s}$ over the past 31 years. A possible decrease in the rate of the period change is not ruled out by the data.

\section{Observational data}

The observational data used in our analysis of the rotation period of HD 37776 is comprised of photometry, spectrophotometry and spectroscopy. A summary is provided in Table 3 and more details are given below.

\subsection{Published photometric data}

We have used almost all of the photometric data of HD 37776 available in the On-line database of photometric observations of $m C P$ stars (Mikulášek et al. 2007a). This is comprised of photometry by Pedersen \& Thomsen (1977), Bartolini et al. (1982), Adelman \& Pyper (1985), Hipparcos (ESA 1997), Adelman (1997), as well as 68 ASAS-3 $V$ measurements acquired in 2002-2006. The ASAS-3 (Pojmański 2002) data are rather noisy (see the corresponding error bar in Fig. 5 below) but are suitable for determining the period. On the other hand, the infrared measurements of Groote \& Kaufmann (1983) have been excluded from our discussion because of their poor phase coverage.

The final collection of published photometric data included in our analysis consists of a total of 1116 measurements carried out in the $u v b y(\beta), U B V, V, H_{\mathrm{p}}$, and $B_{\mathrm{T}} V_{\mathrm{T}}$ photometric systems.

\subsection{New photometric observations}

We obtained two new $U B V$ and $B V$ photometric time series of HD 37776, adding a further 550 measurements. The first set was acquired in 2006 (5 nights) and 2007 (8 nights) in the $U B V$ system at the Hvar Observatory in Croatia with the $0.65 \mathrm{~m}$ reflector equipped with a single-channel photometer and an EMI 9789QB phototube. In 2006 we used HD 36166 (B2 V; $V=9, B-V=$ $-0.198, U-B=-0.843)$ as the comparison star. HD 36591 (B1 IV; $V=5.344, B-V=-0.189, U-B=-0.927$ ) was chosen as the comparison star in 2007. Our $U B V$ observations consisted of 10-second integrations in each filter; data were carefully reduced to the standard $U B V$ system and corrected for differential extinction using the reduction code HEC 22 (Harmanec et al. 1994). The observations are rather noisy because the air mass of HD 37776 was typically $\approx 1.8$; the standard error of the measurements is approximately $0.016 \mathrm{mag}$.

The second set of $B V$ photometry was acquired between October 2006 and March 2007 with the T3 $0.4 \mathrm{~m}$ automatic photoelectric telescope (APT) at Fairborn Observatory. This APT uses a temperature-stabilized EMI 9924B photomultiplier tube to measure photon count rates through Johnson $B$ and $V$ filters. HD 37776 and its comparison stars were measured in the following sequence, termed a group observation: $\mathrm{K}-\mathrm{S}-\mathrm{C}-\mathrm{V}-\mathrm{C}-\mathrm{V}-\mathrm{C}-\mathrm{V}-\mathrm{C}-\mathrm{S}-\mathrm{K}$, where $\mathrm{K}$ is the check star (HD 37984, $V=4.90, B-V=1.14, \mathrm{~K} 1 \mathrm{III}), \mathrm{C}$ is the comparison star (HD 37788, $V=5.93, B-V=0.31$, F0 IV), $\mathrm{V}$ is HD 37776, and S is a sky measurement. Three V-C and two K-C differential magnitudes are formed from each sequence and averaged together to create group means.

Group mean differential magnitudes with internal standard deviations greater than $0.01 \mathrm{mag}$ were rejected to eliminate the observations taken under non-photometric conditions. The 209 surviving group means were corrected for differential extinction with nightly extinction coefficients, transformed to the Johnson system with yearly-mean transformation coefficients, and treated as single observations thereafter. Up to five group observations were acquired each clear night at intervals of approximately two hours. Typical precision of a group-mean differential magnitude is $0.003-0.004 \mathrm{mag}$ for this telescope. Further 
Table 1. Equivalent widths of He I lines measured on CFHT and Ondřejov spectra.

\begin{tabular}{|c|c|c|c|c|c|c|c|c|c|c|c|c|c|}
\hline $\begin{array}{c}\text { HJD } \\
2400000+\end{array}$ & $\varphi_{\mathrm{A}}$ & $\varphi_{\text {III }}$ & 4437 & 4471 & $\begin{array}{c}\text { HJD } \\
2400000+\end{array}$ & $\varphi_{\mathrm{A}}$ & $\varphi_{\text {III }}$ & 4013 & 4120 & 4143 & 4168 & $\begin{array}{c}\text { HJD } \\
2400000+\end{array}$ & $\begin{array}{c}6678 \\
\AA\end{array}$ \\
\hline 46450.854 & 0.955 & 0.270 & 0.241 & 2.78 & 46450.806 & 0.924 & 0.239 & 6.082 & 0.572 & 1.513 & 0.334 & 53818.313 & $(1.31)$ \\
\hline 46451.027 & 0.067 & 0.382 & 0.263 & 2.63 & 46450.946 & 0.015 & 0.330 & 6.545 & 0.565 & 1.696 & 0.275 & 54017.531 & (1.45) \\
\hline 46451.748 & 0.536 & 0.851 & 0.199 & 2.30 & 46451.776 & 0.554 & 0.869 & 5.717 & 0.528 & 1.377 & 0.185 & 54017.577 & (1.38) \\
\hline 46451.829 & 0.588 & 0.903 & 0.184 & 2.22 & 46451.904 & 0.637 & 0.952 & 4.816 & 0.449 & 1.248 & 0.160 & 54084.436 & (1.40) \\
\hline 46451.958 & 0.607 & 0.987 & 0.160 & 1.98 & 46452.016 & 0.710 & 0.025 & 4.318 & 0.432 & 1.079 & 0.120 & 54115.439 & (1.60) \\
\hline 46452.743 & 0.183 & 0.497 & 0.230 & 2.57 & 46452.712 & 0.162 & 0.477 & 5.881 & 0.506 & 1.438 & 0.283 & 54192.310 & (1.39) \\
\hline 46452.903 & 0.286 & 0.601 & 0.238 & 2.46 & 46452.821 & 0.233 & 0.548 & 6.032 & 0.541 & 1.618 & 0.250 & & \\
\hline 46452.981 & 0.337 & 0.652 & 0.262 & 2.64 & 46453.766 & 0.847 & 0.162 & 5.487 & 0.500 & 1.143 & 0.199 & & \\
\hline 46453.711 & 0.812 & 0.127 & 0.139 & 2.23 & 46453.937 & 0.958 & 0.273 & 6.301 & 0.548 & 1.668 & 0.251 & & \\
\hline 46453.881 & 0.922 & 0.237 & 0.207 & 2.45 & 46454.774 & 0.502 & 0.817 & 5.236 & 0.434 & 1.341 & 0.187 & & \\
\hline 46453.937 & 0.976 & 0.273 & 0.250 & 2.62 & & & & & & & & & \\
\hline 46453.983 & 0.988 & 0.303 & 0.253 & 2.63 & & & & & & & & & \\
\hline 46454.012 & 0.007 & 0.322 & 0.249 & 2.64 & & & & & & & & & \\
\hline
\end{tabular}

information on the operation of the APT and the analysis of the data can be found in Henry (1995a,b) and Eaton et al. (2003).

All of our newly obtained photometric data are available through SIMBAD and the On-line database of photometric observations of $m C P$ stars (http: //astro.physics . muni.cz/ mcpod).

\subsection{He I $\lambda 4026$ photometry}

Pedersen \& Thomsen (1977) and Pedersen (1979) published 53 measurements of a photometric index measuring the strength the He I $\lambda 4026$ profile that we have transformed into an equivalent width measurement of the line by means of the relation derived by Vetö et al. (1991).

\subsection{CFHT spectrograms}

Twenty-three Coudé spectra of HD 37776 were obtained by DB in 1986 with the Canada-France-Hawaii Telescope (CFHT), the coudé $f / 8$ spectrograph, and the Reticon detector. Details describing the instrument configuration and data reduction can be found in Bohlender (1988). The signal-to-noise ratio $(S / N)$ of the processed spectra was better than 300 and $R=20000$. Ten spectra covering the wavelength interval from 3990-4175 were used to measure radial velocities of the $\mathrm{H} \delta$ line and equivalent widths of individual $\mathrm{He}$ I lines or blends including $\lambda 4009-26, \lambda 4120, \lambda 4143$, and $\lambda 4168$. Thirteen spectrograms covering the 4395-4580 $\AA$ wavelength region were used for equivalent width measurements of the two He I lines $\lambda 4437$ and $\lambda 4471$.

Equivalent widths of He I lines measured on CFHT ( $\lambda 4437$, 4471, 4009-26, 4120, 4143, and 4168) are given in Table 1. The phase $\varphi_{\mathrm{A}}$ is calculated with Adelman's ephemeris (Eq. (1)), while the cubic phase, $\varphi_{\mathrm{III}}$, is derived from Eq. (18).

\subsection{SAO Zeeman spectrograms}

Thirty-five spectra were obtained with the Main Stellar Spectrograph (MSS) of the $6 \mathrm{~m}$ telescope of the Special Astrophysical Observatory (SAO) using a CCD array and Zeeman analyser. The spectra are $100 \AA$ long and have $S / N \geq$ 200. Twenty-four of the observations were centered at $\lambda 5875$ and eleven at $\lambda 4550$, with $R=19000$ and 15000 , respectively. The spectra are described in detail in Romanyuk et al. (1998) and were processed with the ESO MIDAS and NICE software packages (Knyazev \& Shergin 1995; Kudryavtsev 2000). This set of spectra contains all of the spectra used by Khokhlova et al. (2000) for their Doppler-Zeeman mapping of HD 37776.

The results of measurements of equivalent widths of the He I lines $\lambda 5875$ and $\lambda 4471$ measured on spectra obtained at the SAO are summarised in Table 2. EWR and EWL are the equivalent widths in right and left circularly polarized spectra, respectively. The phase $\varphi_{\mathrm{A}}$ is calculated from Adelman's ephemeris (Eq. (1)), while the cubic phase, $\varphi_{\mathrm{III}}$, is derived from (Eq. (18)).

\subsection{Ondřejov and Rozhen spectrograms}

In addition to the CFHT and SAO data summarized above, we have also obtained eight new CCD spectra of the $\mathrm{H} \alpha$ region of HD 37776 between 2005 October and 2007 April. Six spectra obtained at the $2 \mathrm{~m}$ telescope of the Ondřejov Observatory with $S / N \lesssim 100$ and $R=13000$ were centered on the $\mathrm{H} \alpha$ line. The spectra were reduced with IRAF $^{1}$. Since HD 37776 is located in the nebula IC 432, careful data reduction is required to obtain stellar spectra that are not contaminated with nebular emission. Before the extraction of the spectra, we cleaned the 2D FITS frames with the dcr program written by Pych (2004). This enabled us to subtract the background emission using columns with a width of only one pixel and to obtain a stellar spectrum free of background features from the nebula.

Equivalent widths of $\mathrm{He}$ I $\lambda 6678$ line measured on Ondřejov spectra (see Table 1) were not used for the further discussion because of their poor phase coverage.

Two additional spectra were obtained at the $2 \mathrm{~m}$ telescope of the Rozhen Observatory with $S / N \approx 200$ and $R=26000$ and cover the spectral region $\lambda \lambda 6508-6608 \AA$.

Equivalent widths in all of the above spectra were measured using the EQWREC code of Budaj \& Komžík (2001). The results are given in Tables 1 and 2.

\section{Ephemeris for HD 37776: an increasing period}

The phase shifts among light curves, when phased according to the linear Adelman's ephemeris (Eq. (1)), are clearly illustrated

\footnotetext{
1 IRAF is distributed by the National Optical Astronomy Observatory, which is operated by the Association of Universities for Research in Astronomy (AURA), Inc., under cooperative agreement with the National Science Foundation.
} 
Table 2. Equivalent widths of the He I lines $\lambda 5875$ and $\lambda 4471$ measured on SAO spectra.

\begin{tabular}{|c|c|c|c|c|c|c|c|c|c|c|c|c|c|c|}
\hline $\begin{array}{c}\text { HJD } \\
2400000+\end{array}$ & $\varphi_{\mathrm{A}}$ & $\varphi_{\text {III }}$ & EWR & EWL & $\begin{array}{c}\text { HJD } \\
2400000+\end{array}$ & $\varphi_{\mathrm{A}}$ & $\varphi_{\text {III }}$ & EWR & EWL & $\begin{array}{c}\text { HJD } \\
2400000+\end{array}$ & $\varphi_{\mathrm{A}}$ & $\varphi_{\text {III }}$ & EWR & EWL \\
\hline He I 5875 & & & & & He I 5875 & & & & & $\mathrm{He}$ I 4471 & & & & \\
\hline 49641.469 & 0.567 & 0.824 & - & 1.143 & 50057.486 & 0.940 & 0.184 & 1.267 & 1.071 & 50500.252 & 0.698 & 0.927 & 2.433 & 2.641 \\
\hline 49641.601 & 0.653 & 0.909 & 0.988 & 0.970 & 50057.508 & 0.955 & 0.198 & 1.249 & 1.253 & 50681.558 & 0.531 & 0.753 & 2.660 & 2.376 \\
\hline 49642.425 & 0.188 & 0.445 & 1.238 & 1.255 & 50059.388 & 0.177 & 0.420 & 1.427 & 1.388 & 50707.472 & 0.373 & 0.594 & 3.069 & 2.642 \\
\hline 49736.303 & 0.200 & 0.454 & 1.203 & 1.325 & 50059.412 & 0.192 & 0.436 & 1.360 & 1.264 & 50709.550 & 0.723 & 0.944 & 2.217 & 2.047 \\
\hline 49736.326 & 0.215 & 0.469 & 1.189 & 1.125 & 50060.302 & 0.770 & 0.014 & 0.931 & 0.957 & 50710.490 & 0.334 & 0.555 & 2.365 & 2.773 \\
\hline 49738.262 & 0.474 & 0.727 & 1.413 & 1.248 & 50060.324 & 0.785 & 0.028 & 0.706 & 0.989 & 51799.564 & 0.134 & 0.313 & 2.875 & 2.653 \\
\hline 49788.184 & 0.918 & 0.171 & 1.076 & 1.148 & 50060.524 & 0.915 & 0.158 & 1.137 & 1.120 & 51799.583 & 0.146 & 0.325 & 2.820 & 2.619 \\
\hline 49788.208 & 0.933 & 0.186 & 1.022 & 1.062 & 50060.546 & 0.929 & 0.173 & 1.214 & 1.205 & 52333.158 & 0.922 & 0.079 & 2.184 & 2.184 \\
\hline 50056.527 & 0.317 & 0.561 & 1.303 & 1.181 & 50415.372 & 0.534 & 0.766 & 1.464 & 1.449 & 52333.174 & 0.932 & 0.089 & 2.343 & 2.437 \\
\hline 50056.544 & 0.328 & 0.572 & 1.353 & 1.179 & 50415.397 & 0.550 & 0.782 & 1.478 & 1.460 & 52333.190 & 0.943 & 0.100 & 2.558 & 2.820 \\
\hline 50057.286 & 0.810 & 0.054 & 0.945 & 0.869 & 50415.420 & 0.565 & 0.797 & 1.410 & 1.402 & 52333.220 & 0.962 & 0.119 & 2.469 & 2.616 \\
\hline 50057.308 & 0.824 & 0.068 & 1.056 & 1.065 & 50415.625 & 0.698 & 0.930 & 1.466 & 1.387 & & & & & \\
\hline
\end{tabular}

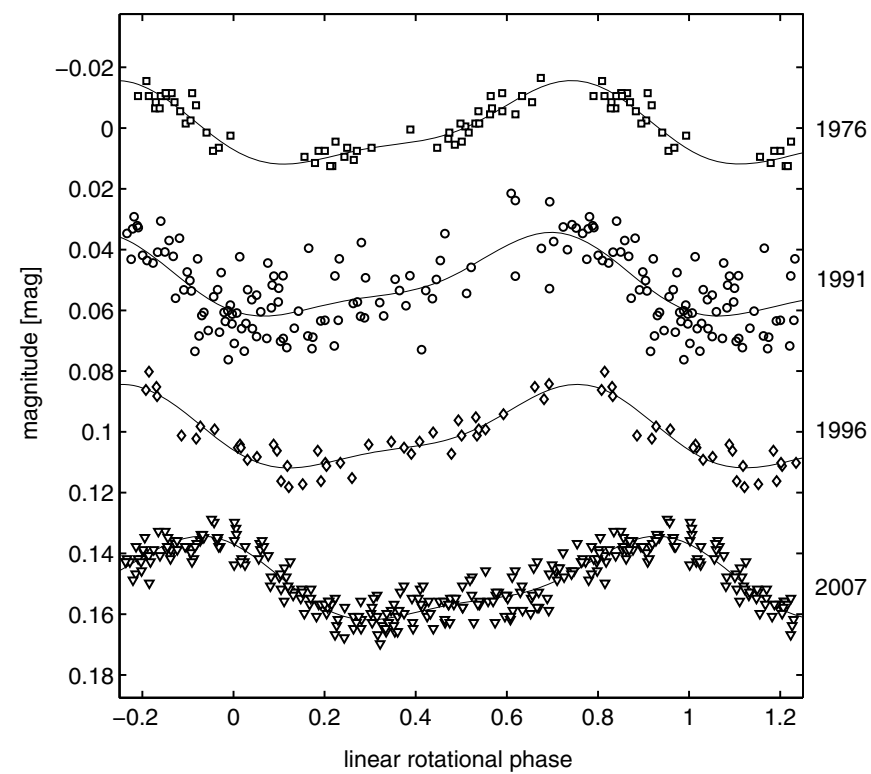

Fig. 1. Observations of HD 37776 obtained in $y, V$, and $H_{\mathrm{p}}$ plotted versus linear phase (see Eq. (1)). Data are taken from Pedersen \& Thomsen (1977), $y$ (ם); Hipparcos (ESA 1997), $H_{\mathrm{p}}(0)$; Adelman (1997), $y(\diamond)$; and T3 APT (this paper), $V(\nabla)$. For clarity the curves have been shifted by $0.05 \mathrm{mag}$ and fitted by the same shifted smooth curve. The year of each set of observations is given on the right side of the figure. Light curves are shifted in the ordinate for clarity. Note the apparent phase shifts of the light curves.

in Fig. 1, which shows separate $y, V$, and $H_{\mathrm{p}}$ light curves obtained for HD 37776 in 1976, 1991, 1996, and 2007. Similar behaviour is exhibited by the spectroscopic observations. The measured equivalent width variations of the $\mathrm{He}$ I lines phased on the same linear ephemeris are presented in Fig. 2. The Pedersen \& Thomsen (1977) and Pedersen (1979) He I 14026 data from 1976 and the SAO data obtained in 1996-2002 have similar phase behaviour (as do the 1976 and 1996 photometry shown in Fig. 1), while the CFHT data obtained in 1986, are apparently shifted with respect to the former data by approximately $0.07 P$.

Time shifts among photometric and spectrophotometric phase curves of HD 37776 are quantified in Table 3. Table columns give the year of observations, the phases of the maxima of the light curves or the minima of the He I equivalent widths corrected by +0.026 in phase according to both the linear (A) and the cubic (III) ephemerides, $\varphi_{\mathrm{A}}^{\mathrm{ext}}$ and $\varphi_{\mathrm{III}}^{\mathrm{ext}}$ (see Eqs. (1) and

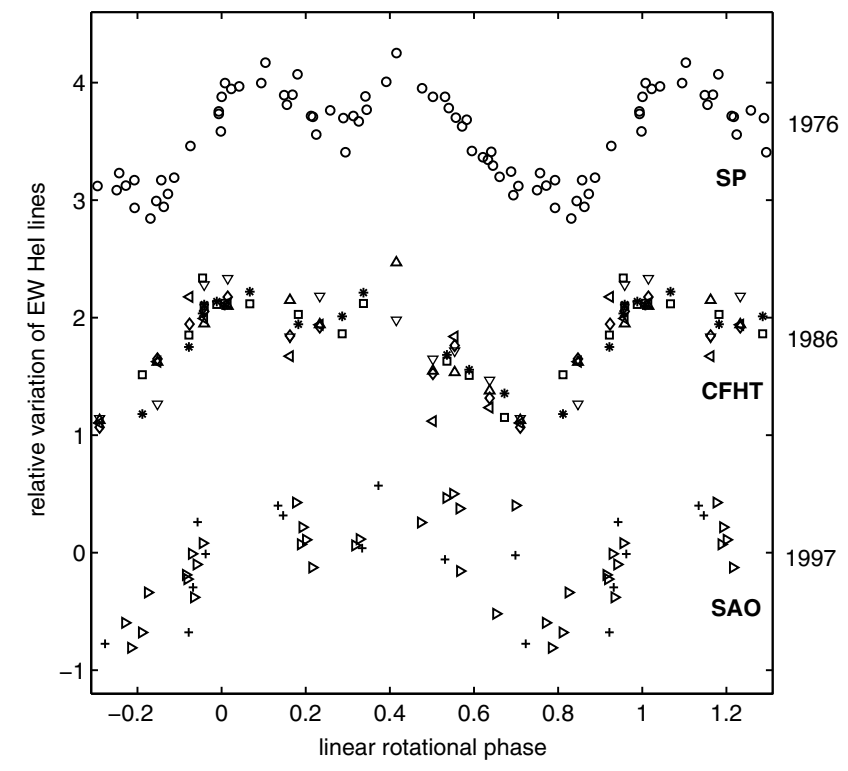

Fig. 2. Relative variations of the equivalent width of He I lines phased according to the ephemeris given in Eq. (1) for Pedersen \& Thomsen (1977) and Pedersen (1979) spectrophotometry of the $\lambda 4026$ line (o); CFHT spectroscopy: $\lambda 4437(\square), \lambda 4471(\diamond), \lambda 4009-26(\triangleleft), \lambda 4120(\nabla)$, $\lambda 4143(\triangle)$, and $\lambda 4168(*)$; SAO spectroscopy: $\lambda 5876(\triangleright)$ and $\lambda 4471(+)$. The Pedersen \& Thomsen (1977) and Pedersen (1979) spectrophotometry was performed in 1976 while the CFHT spectra were obtained in 1986 and the SAO spectra between 1994 and 2002.

(18)), the phase uncertainties, $\delta \varphi$, the number of measurements, $N$, the type of measurements, and the source.

It is apparent that these phase shifts cannot be removed by a simple improvement of the linear ephemeris.

\subsection{Orthogonal cubic ephemeris}

The relative shifts of the light and equivalent width curves of He I lines of HD 37776 during the past 31 years indicate that the period of the star has undergone secular changes that can be described by a smooth function of time $P(t)$. Since we do not observe the change of the period directly, but through the phase shifts of the light and equivalent width curves, we introduce a monotonically growing "phase function" $t(\vartheta)$ and its 
Table 3. Phases of the extrema derived from photometry and spectrophotometry of HD 37776.

\begin{tabular}{crrcrcl}
\hline \hline Year & $\varphi_{\mathrm{A}}^{\text {ext }}$ & $\varphi_{\text {III }}^{\text {ext }}$ & $\delta \varphi$ & $N$ & Data type & Source \\
\hline 1976 & 0.744 & -0.002 & 0.004 & 216 & $u v b y$ & Pedersen \& Thomsen (1977) \\
1976 & 0.748 & 0.006 & 0.007 & 53 & He I $\lambda 4026$ & Pedersen \& Thomsen (1977); Pedersen (1979) \\
1978 & 0.725 & 0.003 & 0.011 & 157 & $U B V$ & Bartolini et al. (1982) \\
1982 & 0.692 & 0.003 & 0.011 & 87 & $u v b y \beta$ & Adelman \& Pyper (1985) \\
1986 & 0.685 & -0.000 & 0.007 & 66 & $E W_{\mathrm{He}}$ & CFHT - this paper \\
1991 & 0.693 & -0.016 & 0.014 & 361 & $B_{\mathrm{T}} V_{\mathrm{T}} H_{\mathrm{p}}$ & ESA (1997) \\
1996 & 0.751 & 0.000 & 0.005 & 168 & $u v b y$ & Adelman (1997) \\
1997 & 0.756 & -0.015 & 0.011 & 69 & $E W_{\mathrm{He}}$ & SAO - this paper \\
2004 & 0.895 & 0.017 & 0.024 & 168 & $V$ & Pojmański (2002) \\
2007 & 0.919 & -0.001 & 0.004 & 550 & $(U) B V$ & Hvar \& T3 APT photometry - this paper \\
\hline
\end{tabular}

inverse function $\vartheta(t)$. There is a trivial relation between the phase function and the instantaneous period $P(\vartheta)$

$$
\frac{\mathrm{d} t(\vartheta)}{\mathrm{d} \vartheta}=P(\vartheta), \Longrightarrow t(\vartheta)=\int_{\theta=0}^{\vartheta} P(\theta) \mathrm{d} \theta, \quad \vartheta(t)=\int_{\tau=0}^{t} \frac{\mathrm{d} \tau}{P(\tau)}
$$

We put the origin of the phase function near the weighted average of times of the observations. Now we can approximate the development of the instantaneous period $P(t)$ at $t=M_{0}$ by the first three terms of its Taylor expansion:

$P(t)=P_{0}+\dot{P}_{0}\left(t-M_{0}\right)+\frac{1}{2} \ddot{P}_{0}\left(t-M_{0}\right)^{2}$,

where $M_{0}, P_{0}, \dot{P}_{0}$, and $\ddot{P}_{0}$ are the heliocentric Julian date (HJD) of the origin, the period at $t=M_{0}$, and first and second time derivatives of the period at $t=M_{0}$ respectively. Using Eq. (2) we can write for the phase function $t(\vartheta)$ :

$t(\vartheta)=M_{0}+P_{0} \vartheta+\frac{1}{2} P_{0} \dot{P}_{0} \vartheta^{2}+\frac{1}{6} P_{0}^{2} \ddot{P}_{0} \vartheta^{3}$.

Substituting Eq. (3) in the last expression in Eq. (2) and assuming $\dot{P}^{2} \ll \ddot{P} P_{0}$ we can approximate the corresponding inversion phase function $\vartheta(t)$ by polynomials

$\vartheta(t)=\vartheta_{1}-\frac{1}{2} \dot{P}_{0} \vartheta_{1}^{2}-\frac{1}{6} P_{0} \ddot{P}_{0} \vartheta_{1}^{3} ; \quad \vartheta_{1}(t)=\frac{t-M_{0}}{P_{0}}$,

where $\vartheta_{1}(t)$ is an auxiliary linear function of time.

It is advantageous to switch from standard polynomials to orthogonal ones. Then Eqs. (4) and (5) transform into

$$
\begin{aligned}
t(\vartheta) \cong & \tilde{M}+\tilde{P} \vartheta+\frac{1}{2} \tilde{P} \tilde{P}^{\prime}\left(\vartheta^{2}-\alpha_{1} \vartheta-\alpha_{2}\right) \\
& +\frac{1}{6} \tilde{P}^{2} \tilde{P}^{\prime \prime}\left(\vartheta^{3}-\alpha_{3} \vartheta^{2}-\alpha_{4} \vartheta-\alpha_{5}\right), \text { and } \\
\vartheta(t) \cong & \vartheta_{\mathrm{I}}-\frac{1}{2} \tilde{P}^{\prime}\left(\vartheta_{\mathrm{I}}^{2}-\alpha_{1} \vartheta_{\mathrm{I}}-\alpha_{2}\right) \\
& -\frac{1}{6} \tilde{P} \tilde{P}^{\prime \prime}\left(\vartheta_{\mathrm{I}}^{3}-\alpha_{3} \vartheta_{\mathrm{I}}^{2}-\alpha_{4} \vartheta_{\mathrm{I}}-\alpha_{5}\right) ; \quad \vartheta_{\mathrm{I}}(t)=\frac{t-\tilde{M}}{\tilde{P}},
\end{aligned}
$$

where $\tilde{M}, \tilde{P}, \tilde{P}^{\prime}$, and $\tilde{P}^{\prime \prime}$ have the analogous meaning of the heliocentric Julian date (HJD) of the basic extremum, the mean period, and the mean first and second time derivatives of the period. $\vartheta_{\mathrm{I}}(t)$ is the inverse phase function for the linear fit. The constants $\alpha_{1}, \alpha_{2}, \alpha_{3}, \alpha_{4}$, and $\alpha_{5}$ are determined so that terms in Eqs. (6) and (7) are mutually orthogonal on the set of data $\left\{\vartheta_{i}, w_{i}\right\}$, where $w_{i}$ is the intrinsic weight of the $i$ th data point. The weighted mean of the $p$ th order of $\vartheta, \overline{\vartheta^{p}}$ then is:

$\overline{\vartheta^{p}}=\frac{\sum_{i=1}^{N} \vartheta_{i}^{p} w_{i}}{\sum_{i=1}^{N} w_{i}}$.
Assuming $\bar{\vartheta}=0$ we can set five conditions of orthogonality with five unknown parameters in the form:

$$
\begin{aligned}
& \overline{\left(\vartheta^{2}-\alpha_{1} \vartheta-\alpha_{2}\right)}=0 ; \quad \overline{\left(\vartheta^{3}-\alpha_{3} \vartheta^{2}-\alpha_{4} \vartheta-\alpha_{5}\right)}=0 ; \\
& \overline{\vartheta\left(\vartheta^{2}-\alpha_{1} \vartheta-\alpha_{2}\right)}=0 ; \quad \overline{\vartheta\left(\vartheta^{3}-\alpha_{3} \vartheta^{2}-\alpha_{4} \vartheta-\alpha_{5}\right)}=0 ; \\
& \overline{\left(\vartheta^{2}-\alpha_{1} \vartheta-\alpha_{2}\right)\left(\vartheta^{3}-\alpha_{3} \vartheta^{2}-\alpha_{4} \vartheta-\alpha_{5}\right)}=0 .
\end{aligned}
$$

After some algebra we find

$$
\begin{aligned}
& \alpha_{1}=\frac{\overline{\vartheta^{3}}}{\overline{\vartheta^{2}}}, \quad \alpha_{2}=\overline{\vartheta^{2}}, \quad \alpha_{3}=\frac{{\overline{\vartheta^{2}}}^{2} \overline{\vartheta^{3}}+\overline{\vartheta^{3}} \overline{\vartheta^{4}}-\overline{\vartheta^{2}} \overline{\vartheta^{5}}}{{\overline{\vartheta^{2}}}^{3}+{\overline{\vartheta^{3}}}^{2}-\overline{\vartheta^{2}} \overline{\vartheta^{4}}} \\
& \alpha_{4}=\frac{\overline{\vartheta^{3}} \overline{\vartheta^{5}}+{\overline{\vartheta^{2}}}^{2} \overline{\vartheta^{4}}-{\overline{\vartheta^{4}}}^{2}-{\overline{\vartheta^{3}}}^{2} \overline{\vartheta^{2}}}{{\overline{\vartheta^{2}}}^{3}+{\overline{\vartheta^{3}}}^{2}-\overline{\vartheta^{2}} \overline{\vartheta^{4}}} \\
& \alpha_{5}=\frac{{\overline{\vartheta^{2}}}^{2} \bar{\vartheta}^{5}+{\overline{\vartheta^{3}}}^{3}-2 \overline{\vartheta^{3}} \overline{\vartheta^{4}}}{{\overline{\vartheta^{2}}}^{3}+{\overline{\vartheta^{3}}}^{2}-\overline{\vartheta^{2}} \overline{\vartheta^{4}}}
\end{aligned}
$$

The constants $\alpha_{1}, \alpha_{2}, \alpha_{3}, \alpha_{4}$, and $\alpha_{5}$ quantify the phase coverage of the data over the entire observation period. If the data points were distributed evenly over the entire observational interval then $\alpha_{1}=\alpha_{3}=\alpha_{5}=0, \alpha_{4}=\overline{\vartheta^{4}} / \overline{\vartheta^{2}}$, and $\alpha_{2}=\overline{\vartheta^{2}}$.

The use of orthogonal polynomials (for details see Mikulášek 2007) for the ephemerides of variable stars is highly recommended as it enables an estimate of the true uncertainties for all derived parameters, an assessment of the importance of individual terms of the regression model and a calculation of the true uncertainties of any predictions. It also provides a swift transition from a higher degree of approximation to a lower one; for example, neglecting the last term in Eq. (6) or Eq. (7) we obtain the quadratic ephemeris, while neglecting the two last terms we get a linear ephemeris.

\subsection{Regression model}

Based on the similarity of all of the phase curves we developed special robust codes for analysis of the data. We were able to describe the observed phase curves with a minimum of parameters, while the robustness of the method allows processing of data with many outliers as occurs, for example, with the ASAS data.

All of the photometric data summarized in Table 3 were sorted into nine groups of so-called "colours" according to their effective wavelength and the width of the photometric band: $u, U, v, B$ and $B_{\mathrm{T}}, b, H_{\mathrm{p}}, y, V$ and $V_{\mathrm{T}}$, and the index $\beta$. We assumed that the light curves within a distinct "colour" have the same amplitude and, eventually, the same phase shift. In a similar way we also introduced spectroscopic colours: a "colour" for the equivalent widths of each of the nine measured He I lines. 
Both the photometric and spectroscopic observations were fitted by the following general model

$y_{c j}\left(t_{i}\right) \sim \bar{y}_{c j}+A_{\mathrm{c}} F\left[\varphi\left(t_{i}\right), \boldsymbol{\beta}\right] ; \quad \varphi\left(t_{i}\right)=\operatorname{Frac}\left(\vartheta_{i}-\Delta \varphi_{\mathrm{c}}\right)$,

where $y_{c j}\left(t_{i}\right)$ is the magnitude observed in the colour $c$ (or the equivalent width of the particular He I line) at the heliocentric time $t_{i}$ by the $j$ th observer, $\bar{y}_{c j}$ is the mean value of the $c$ colour for the $j$ th observer, $A_{\mathrm{c}}$ is the amplitude in the particular $c$ colour related to the basic model function $F\left[\varphi\left(t_{i}\right), \beta\right]$, which is a function of the phase $\varphi\left(t_{i}\right)$ of the $i$ th measurement and the set of parameters $\beta$ characterizing the form of the basic function $F$. The term $\Delta \varphi_{\mathrm{c}}$ is the colour dependent correction expressing the relative shift of the particular light curve when compared to the others. This correction is significant only in the colours $u$ and possibly $v$, and for the remaining colours it is set to zero.

We found that the simplest basic function with a maximum at phase $\varphi=0$ that satisfactorily describes the shapes of the observed light curves of HD 37776 is one determined by only two parameters $\beta_{1}$ and $\beta_{2}$

$F(\varphi)=\cos (2 \pi \varphi)+\beta_{1} \cos (4 \pi \varphi)+\beta_{2}\left[\sin (2 \pi \varphi)-\frac{1}{2} \sin (4 \pi \varphi)\right]$.

The basic shape of the helium equivalent width phase curves is more or less symmetric and consequently can be described by a function of a single parameter $\beta_{3}$

$F(\varphi)=\cos (2 \pi \varphi)+\beta_{3} \cos (4 \pi \varphi)$.

The incorporation of the He I equivalent width measurements in the analysis of period changes is limited by the possibility of reliably determining phase shifts between the light and equivalent width curves. Fortunately, the He I spectrophotometry of Pedersen \& Thomsen (1977) and Pedersen (1979) was performed in practically the same period of time (1976) as the Pedersen \& Thomsen (1977) uvby photometry, as was the SAO spectroscopy and the Adelman (1997) uvby photometry (1997, see Table 3 ). We find that in both of these cases a sharp minimum of the equivalent width curve precedes the light curve maximum by $\Delta \varphi_{\mathrm{He}}=0.026(5) P$. Using this fact, and assuming there are no long-term changes in the shape of the equivalent width curve (see discussion below), we can then also use the high $S / N$ CFHT spectroscopy, obtained at a time when no photometric observations were made to extract additional phase information.

\subsection{Robust regression procedure}

Eleven observers or observing teams obtained 34 sets of measurements carried out in nine photometric colours and nine different He I lines (additional "colours") giving $N=1895$ individual measurements of various types. To describe their time variation we need a total of 61 free parameters: four parameters for the cubic ephemeris $\left(\tilde{M}, \tilde{P}, \tilde{P}^{\prime}, \tilde{P}^{\prime \prime} ;\right.$ Eqs. (17) and (18)), $9+9$ amplitudes $\left(A_{\mathrm{c}}\right), 34$ mean values of individual phase curves $\left(\bar{y}_{c j}\right)$, two phase shifts for the $u$ and $v$ curves $\left(\Delta \varphi_{u}, \Delta \varphi_{v}\right.$; Eq. (11)), and finally, the three parameters $\beta_{1}, \beta_{2}$, and $\beta_{3}$ (Eqs. (12) and (13)). Values for each of these parameters were determined by the method of iterative minimization of the quantity $S$, a nonlinear function of the parameters $\left\{p_{k}\right\}$

$S(\boldsymbol{p})=\sum_{i=1}^{N}\left[y_{i}-A_{\mathrm{c}} F\left(t_{i}, \boldsymbol{\beta}\right)-\bar{y}_{c j}\right]^{2} w_{i}, \quad \frac{\partial S(\boldsymbol{p})}{\partial \boldsymbol{p}}=\mathbf{0}$.

The common gradient method is a straightforward way of finding the 61 unknown free parameters by means of the nonlinear
LSM regression with known weights, $w_{i}$, of individual measurements.

In observational data one commonly encounters "outliers", data points found relatively far from the others. For the purpose of exploiting the outliers we introduced "robust weights" (Mikulášek et al. 2003) as follows

$w_{i} \propto \sigma_{c j}^{-2} \exp \left[-\left(\frac{\Delta y_{i}}{2.5 \sigma_{c j}}\right)^{4}\right], \sigma_{c j}^{2} \cong 1.23 \frac{\sum_{k=1}^{n_{c j}}\left(\Delta y_{k}\right)^{2} w_{k}}{\sum_{k=1}^{n_{c j}} w_{k}}$

The iterative minimization performed with robust weights is more computationally intensive since the weights change for each successive iteration, but the effect of the outliers is correctly minimized and therefore warrants the longer computing time required.

\subsection{Results}

The results we arrived at after several tens of iterations are as follows: the behaviour of the light curves is described by Eq. (12) with parameters $\beta_{1}=0.318(15)$ and $\beta_{2}=-0.209(17)$. The equivalent width curve of $\mathrm{He} \mathrm{I}$ is best represented with $\beta_{3}=0.75(5)$ in Eq. (13). The shifts of the $u$ and $v$ curves with respect to the remaining light curves are given by $\Delta \varphi_{u}=-0.030(5)$ and $\Delta \varphi_{v}=0.010(5)$. The coefficients $\alpha_{i}$ in Eq. (10) are $\alpha_{1}=$ $-580, \alpha_{2}=1.03 \times 10^{7}, \alpha_{3}=-170, \alpha_{4}=1.3 \times 10^{7}$ and $\alpha_{5}=$ $-4.2 \times 10^{9}$. Furthermore, we find

$$
\begin{array}{llrl}
\tilde{M} & =2448857.1239(34), & & \tilde{P}=1.5387115(9), \\
\tilde{P}^{\prime} & =1.72(6) \times 10^{-8}, & & \tilde{P}^{\prime \prime}=-29(13) \times 10^{-13} \mathrm{~d}^{-1},
\end{array}
$$

whereas $\tilde{P}^{\prime \prime}=\ddot{P}(0)=\bar{P}$.

After substitution in Eqs. (6) and (7) we then find for the HJD of the photometric maximum $T_{\max }$ corresponding to the epoch $E$

$$
\begin{aligned}
T_{\max }(E)= & 2448857.1239(34)+1.5387115(9) \times E \\
& +1.32(5) \times\left(E^{\prime 2}+0.058 E^{\prime}-0.103\right) \\
& -1.1(5) \times\left(E^{\prime 3}+0.017 E^{\prime 2}-0.13 E^{\prime}+0.0042\right)
\end{aligned}
$$

where $E^{\prime}=10^{-4} E$, and

$$
\begin{aligned}
\vartheta(T)= & \vartheta_{\mathrm{I}}-0.859(33) \times\left(\vartheta_{\mathrm{I}}^{\prime 2}+0.058 \vartheta_{\mathrm{I}}^{\prime}-0.103\right) \\
& +0.74(35) \times\left(\vartheta_{\mathrm{I}}^{\prime 3}+0.017 \vartheta_{\mathrm{I}}^{\prime 2}-0.13 \vartheta_{\mathrm{I}}^{\prime}+0.0042\right), \quad(18) \\
\vartheta_{\mathrm{I}}= & 0.6498944(4) \times[T-2448857.1239(34)], \vartheta_{\mathrm{I}}^{\prime}=10^{-4} \vartheta_{\mathrm{I}}
\end{aligned}
$$

where $T$ is an arbitrarily chosen HJD and $\vartheta(T)$ is the corresponding value of the phase function at that instant in time. Subsequently we come at numerical values

$M_{0}=\tilde{M}-\frac{1}{2} \tilde{P}^{\prime} \alpha_{2}-\frac{1}{6} \tilde{P}^{2} \tilde{P}^{\prime \prime} \alpha_{5}=2448857.031(5)$,

$P_{0}=\tilde{P}\left(1-\frac{1}{2} \tilde{P}^{\prime} \alpha_{1}-\frac{1}{6} \tilde{P} \tilde{P}^{\prime \prime} \alpha_{4}\right)=1.538734(7)$,

$\bar{P}=\tilde{P}\left[1-\frac{1}{2} \tilde{P}^{\prime} \alpha_{1}-\frac{1}{6} \tilde{P} \tilde{P}^{\prime \prime}\left(\alpha_{4}-3 \alpha_{2}\right)\right]=1.538699(9)$,

$\overline{\dot{P}} \cong \dot{P}(0) \cong \tilde{P}^{\prime}-\frac{1}{3} \tilde{P} \tilde{P}^{\prime \prime} \alpha_{3}=1.69(7) \times 10^{-8}$, and

$\overline{\dot{P} / P} \doteq \overline{\dot{P}} / \tilde{P}=4.01(17) \times 10^{-6}$ year $^{-1}$,

where $M_{0}$ is the HJD of the basic maximum, $P_{0}$ is the instantaneous period and $\dot{P}(0)$ its instantaneous derivative at the moment $M_{0}, \bar{P}$ is the mean period and $\overline{\dot{P}}$ is the mean value of the derivative of the period.

Figure 3 illustrates the light variations of HD 37776 plotted versus the cubic ephemeris (Eq. (18)). All colour variations show 


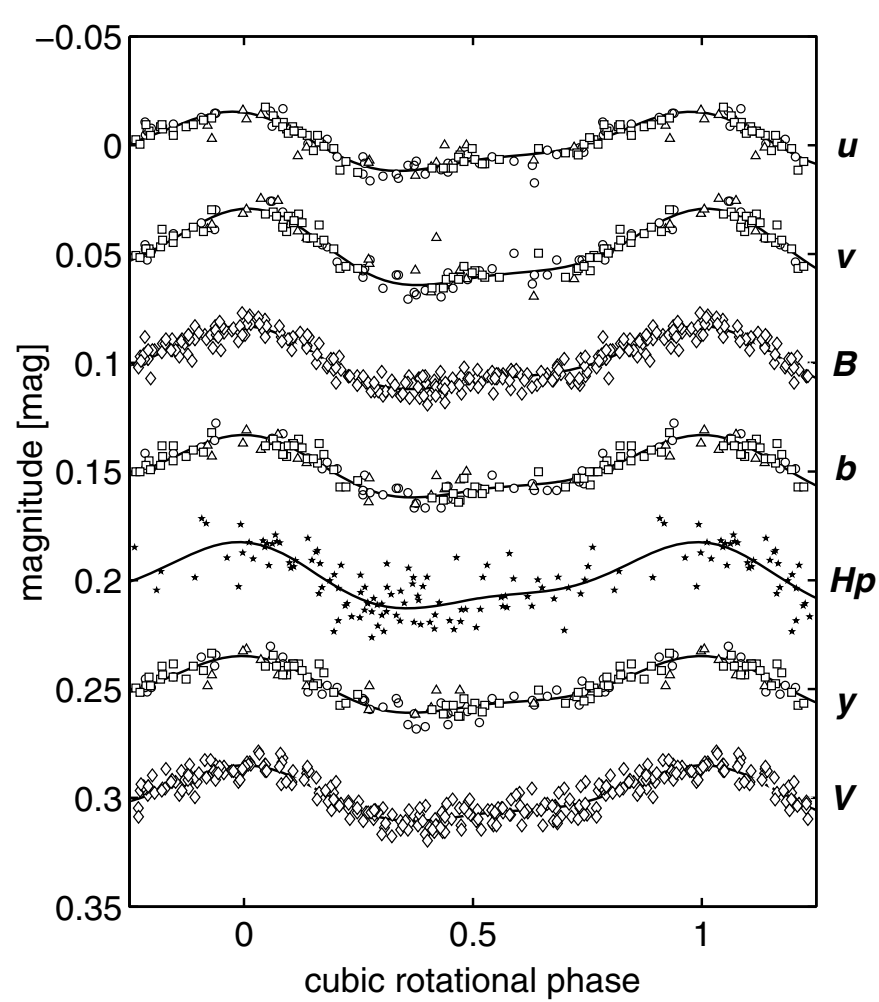

Fig. 3. Photometric observations of HD 37776 plotted as a function of the cubic phase (see Eq. (18)). Data are from Pedersen \& Thomsen

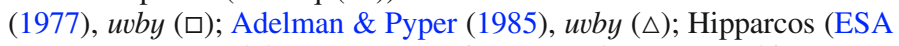
1997), $H_{\mathrm{p}}(\star)$; Adelman (1997) uvby (o); and T3 APT (this paper), $B V(\diamond)$.

a common phasing and demonstrate that this new ephemeris describes the observed light variations over the last 31 years very well. We discuss the nature of the period change this implies in Sect. 4.1.

\subsection{Long-term stability of the light curves of HD 37776}

The shapes of the light curves of the majority of well-studied $\mathrm{mCP}$ stars do not appear to change on a scale of several tens of years. This is a consequence of the usual persistence of spectroscopic and photometric spots on the surfaces of the mCP stars. It seems very likely that both the amplitude and the shape of the observed light curves of HD 37776 remain constant. We demonstrate this qualitatively in Fig. 4 where we show $y$ and $V$ light curves obtained in 1976, 1996 and 2007. The light curves seem to be, within observational errors, identical. However, Pyper et al. (1998), Žižňovský et al. (2000) and Adelman et al. (2001) have demonstrated that in a few mCp stars, namely CU Vir and 56 Ari, a change in the period of the star is accompanied by a change in the amplitude and shape of the light curve. We have therefore performed a more quantitative examination of the possibility of long-term changes in the light curves of HD 37776.

A significant disadvantage of the HD 37776 photometric material is the inhomogeneity of the photometric systems in which the star was observed during the 1976-2007 period. Hence we are not able to study the time development of the shapes of the light curves in a single colour directly. Instead we make a quantitative estimate of the possible changes in amplitudes and shapes

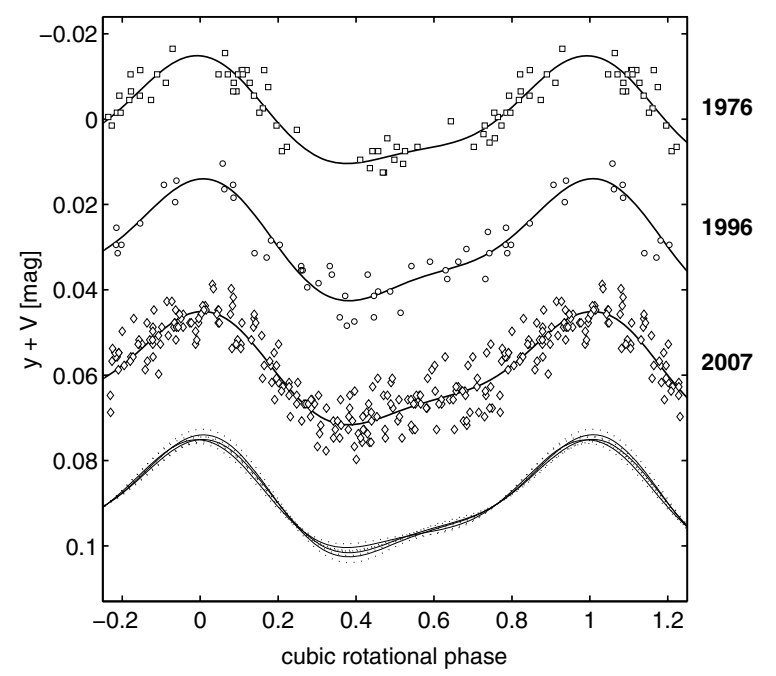

Fig. 4. $V$ and $y$ light curves obtained over 31 years folded in the cubic ephemeris. The light curves were fitted separately for each observational period by the standard weighted LSM technique. At the bottom of the figure all of the fits are plotted together to illustrate the stability of the shape of the seasonal curves. The dotted lines surrounding the curves represent the uncertainties of the fits.

of the star's "colour" curves by expanding the model described by Eqs. (11) and (12) as follows

$m(\vartheta) \sim \bar{m}_{c j}+\left(1+\beta_{4} E\right) A\left(\lambda_{\mathrm{c}}\right) F(\varphi), \quad A\left(\lambda_{u}\right)=A_{u} ;$

if $c \neq u, U: A\left(\lambda_{\mathrm{c}}\right)=\bar{A}\left[1+a_{1}\left(\lambda_{\mathrm{c}}-\gamma_{1}\right)+a_{2}\left(\lambda_{\mathrm{c}}^{2}-\gamma_{2} \lambda_{\mathrm{c}}-\gamma_{3}\right)\right]$,

$\gamma_{1}=\bar{\lambda} ; \quad \gamma_{2}=\frac{\overline{\lambda^{3}}-\overline{\lambda^{2}} \bar{\lambda}}{\overline{\lambda^{2}}-\bar{\lambda}^{2}} ; \quad \gamma_{3}=\frac{{\overline{\lambda^{2}}}^{2}-\overline{\lambda^{3}} \bar{\lambda}}{\overline{\lambda^{2}}-\bar{\lambda}^{2}}$,

where

$$
\begin{aligned}
F(\vartheta, \boldsymbol{\beta})= & \cos (2 \pi \varphi)+\left(1+\beta_{5} \vartheta\right) \beta_{1} \cos (4 \pi \varphi) \\
& +\left(1+\beta_{6} \vartheta\right) \beta_{2}\left[\sin (2 \pi \varphi)-\frac{1}{2} \sin (4 \pi \varphi)\right] .
\end{aligned}
$$

The new coefficients $\beta_{4}, \beta_{5}, \beta_{6}$ allow for the possible secular relative change in the amplitude and shape of a light curve. Again, using all of the photometric data and adopting the cubic ephemeris we find that the values of these three coefficients differ from zero by an amount less than their relative uncertainty: $\beta_{4}=(0.10 \pm 0.12) \%$ year $^{-1}, \beta_{5}=(-0.3 \pm 0.4) \%$ year $^{-1}$, and $\beta_{6}=$ $(0.5 \pm 0.6) \%$ year $^{-1}$.

Consequently, this confirms that there is no evidence for secular variations in the shapes of HD 37776's light curves during the last 31 years.

\section{Discussion}

\subsection{The nature of the observed period changes}

The seasonal phases of the maxima of the light and equivalent width curves of HD 37776 determined for the individual sets of observations derived using the linear ephemeris (Eq. (1)) are shown in Fig. 5. The parabolic shape of the curve defined by the data points in the diagram indicates a gradual lengthening of the period of the spectroscopic and photometric variations of the star over the 31-year history of the observations discussed above. The best approximation to the model points is a cubic parabola, although a quadratic fit is also possible.

The precisely measured shift of the 1986 CFHT He I equivalent width curve rules out the fit by the two straight line segments 


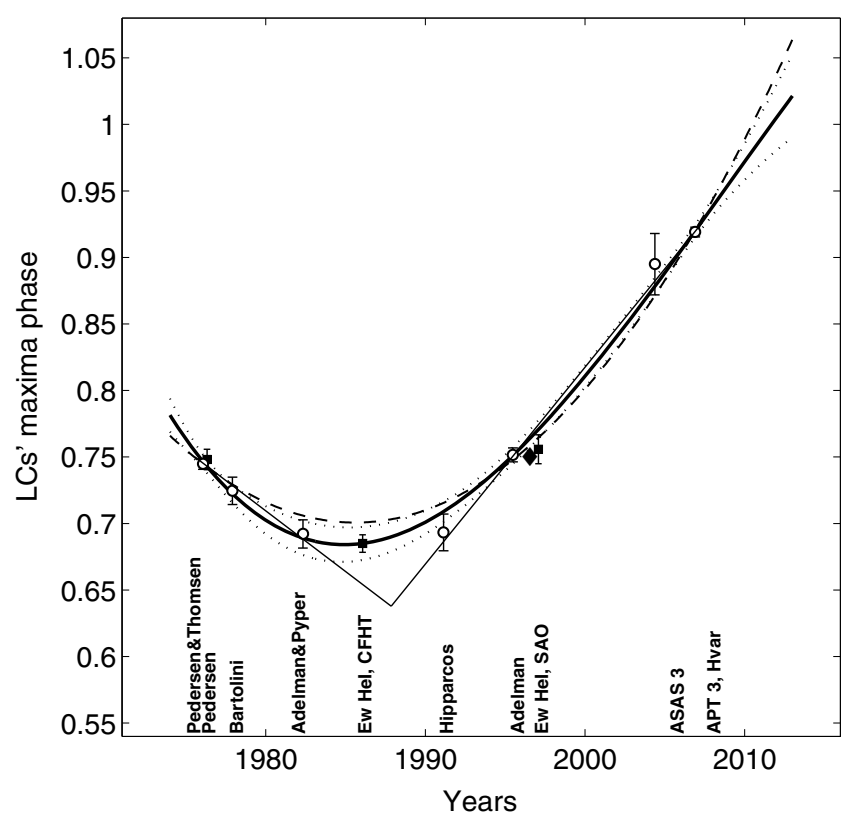

Fig. 5. The phase of the maxima of the light curves of HD 37776 as a function of time. Measurements derived from photometry are denoted by open circles. Filled squares correspond to the predicted phases of light maxima derived from the corresponding minima of the phase curves of the equivalent widths of $\mathrm{He}$ I lines (see Fig. 2) and corrected by $0.026 P$ as described in the text. The filled diamond corresponds to the light curves predicted by Krtička et al. (2007) from the surface abundance maps of Khokhlova et al. (2000). The dashed and solid curves correspond to the quadratic and cubic ephemerides, respectively, while the dotted lines indicate the uncertainty of the fit. The two straight line segments show the phase dependence if it is assumed that the period of HD 37776 changed abruptly by $13.5 \pm 0.9 \mathrm{~s}$ in 1988 .

shown in Fig. 5, which correspond to an abrupt period change (as found for CU Vir; Pyper et al. 1998) of $13.5 \pm 0.9 \mathrm{~s}$ around 1988.

Using the cubic orthogonal model defined in the foregoing Sect. 3 we can directly calculate the time dependence of the period $P(\vartheta)$ on the phase function $\vartheta$ including its uncertainty:

$P=\frac{\mathrm{d} t}{\mathrm{~d} \vartheta}=\tilde{P}+\tilde{P} \tilde{P}^{\prime}\left(\vartheta-\frac{\alpha_{1}}{2}\right)+\tilde{P}^{2} \tilde{P}^{\prime \prime}\left(\frac{\vartheta^{2}}{2}-\frac{\alpha_{3} \vartheta}{3}-\frac{\alpha_{4}}{6}\right)$.

It follows from Eq. (22) that from 1976 until now the period of the HD 37776 has increased by $17.7 \pm 0.7 \mathrm{~s}$ (or in the case of a quadratic fit, $16.9 \pm 0.6 \mathrm{~s}$ ). The change in the observed period as a function of time is depicted schematically in Fig. 6. The rate of the period increase is extremely high. If we interpret it as the consequence of rotational braking then over the last 31 years the star has slowed by $\frac{1}{2} \tilde{P}^{\prime}\left(E_{\mathrm{e}}-E_{\mathrm{b}}\right)^{2}=0.48($ !) of one stellar revolution, where $E_{\mathrm{e}}-E_{\mathrm{b}}$ is the total number of epochs during the observing period.

We discuss possible causes of the observed period change in detail in the following sections. These include the possibility of i) a light-time effect; ii) free-body precession; iii) evolutionary changes; iv) magnetic braking; and v) angular momentum loss via a continuous/discrete/transient stellar wind outflow.

\subsection{Light-time effect}

Figure 5 evokes comparison to an $\mathrm{O}-\mathrm{C}$ diagram that shows the orbital period variation due to a third body present in a binary star, the so called light-time effect. The rotational period of a star in a simple binary could also be affected in a similar way.

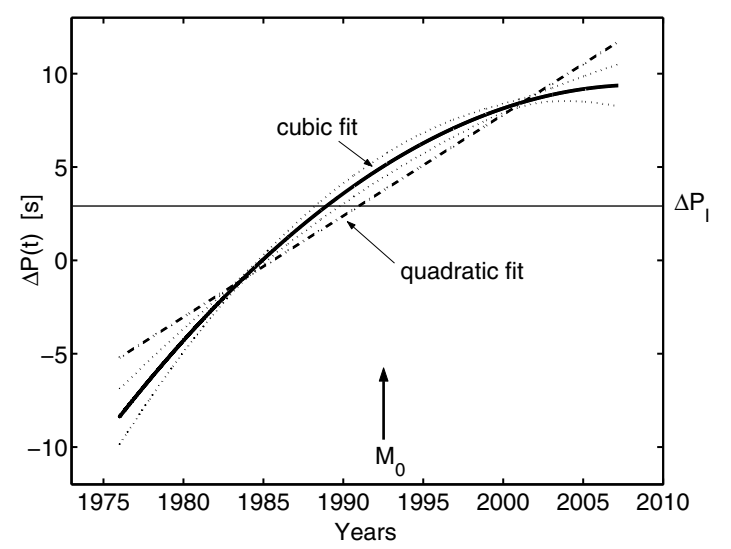

Fig. 6. The progress of the instantaneous period $P(t)$ relative to the Adelman's period $P_{\mathrm{A}}$ (see Eq. (22)) in s. The dashed-dot line corresponds to a quadratic fit and the solid line to a cubic one. The dotted lines enclose the uncertainty of the fit. $M_{0} \cong \tilde{M}$ corresponds to the origin of counting of the phase function, $\Delta P_{\mathrm{I}}$ is given by the relation: $\Delta P_{\mathrm{I}}=\tilde{P}-P_{\mathrm{A}}$.

Given the magnitude and duration of the period change seen in the figure, we estimate that the mass of the secondary component in such a binary would have to be at least 1.28 times higher than that of HD 37776. Only a black hole could contain such a mass and not be seen in the photometry or in the spectrum of the system. The presence of such a companion might then only reveal itself through variations in the radial velocity of at least $26 \mathrm{~km} \mathrm{~s}^{-1}$ over the span of several decades. We have therefore checked published radial velocity values available for HD 37776 and measured additional values in the spectra discussed above.

There are four sources of historical radial velocity measurements for HD 37776. Neubauer (1943) gives $25.5 \pm 2.6 \mathrm{~km} \mathrm{~s}^{-1}$ averaged from seven plates obtained between 1933 and 1942 but without any reference to the date of the individual observations. Wilson \& Joy (1950) give $29 \mathrm{~km} \mathrm{~s}^{-1}$ and the GCRV (Duflot et al. 1995) gives $27 \mathrm{~km} \mathrm{~s}^{-1}$ from ten measurement with no variability, again without dates of the observations. Abt (1970) provides three old Mount Wilson measurements between 16.4 and $35.4 \mathrm{~km} \mathrm{~s}^{-1}$.

We have obtained new radial velocity measurements from our six Ondřejov, two Rozhen and ten CFHT spectra. For these measurements we used only the central portions $(\approx 8 \AA)$ of the $\mathrm{H} \alpha$ and $\mathrm{H} \delta$ Balmer lines to measure radial velocities by applying the CCF technique described by Zverko et al. (2007). The Balmer lines were chosen because hydrogen is the only chemical element with sufficiently strong spectral lines that is spread relatively uniformly over the surface of the star. All radial velocity measurements are summarized in Table 4. Phases $\varphi_{\mathrm{A}}$ and $\varphi_{\mathrm{III}}$ correspond to those calculated with Eqs. (1) and (18), respectively.

These "hydrogen" radial velocities measured from spectra obtained in the last twenty-one years are nearly constant with $\overline{R V}=33.1(9) \mathrm{km} \mathrm{s}^{-1}$. Only the earliest value of Abt (1970) disagrees with the recent data. The mean year-to-year change we found is almost zero, $a_{\mathrm{obs}}=-0.16(9) \mathrm{km} \mathrm{s}^{-1} \mathrm{year}^{-1}$, while the light time hypothesis predicts acceleration of $a \simeq c \overline{\dot{P}} / P=$ $1.20(5) \mathrm{km} \mathrm{s}^{-1}$ year $^{-1}$, where $c$ is the speed of light.

Consequently, we conclude that the observed $\mathrm{O}-\mathrm{C}$ changes in the light curve of HD 37776 cannot be attributed to the presence of an unseen, massive companion and therefore the observed period increase is not the result of a "light-time effect". 
Table 4. List of radial velocity measurements.

\begin{tabular}{|c|c|c|c|c|c|}
\hline Year & $\begin{array}{c}\text { HJD } \\
+2400000\end{array}$ & $\varphi_{\mathrm{A}}$ & $\varphi_{\mathrm{III}}$ & $\begin{array}{c}\mathrm{RV} \quad \delta \mathrm{RV} \\
\mathrm{km} \mathrm{s}^{-1}\end{array}$ & Source \\
\hline 1920 & 22648.964 & & & $16.4 \quad 1.8$ & Abt (1970) \\
\hline 1921 & 22746.735 & & & 35.4 & \\
\hline 1921 & 23015.851 & & & 32.6 & \\
\hline \multirow[t]{3}{*}{$1933-42$} & & & & 25.52 .6 & Neubauer (1943) \\
\hline & & & & $29 \quad 3.4$ & Wilson \&Joy (1950) \\
\hline & & & & 27 & GCRV \\
\hline 1986 & 46450.806 & 0.924 & 0.239 & $\begin{array}{ll}37.6 & 1.1\end{array}$ & CFHT \\
\hline 1986 & 46450.946 & 0.015 & 0.330 & $26.6 \quad 0.9$ & \\
\hline 1986 & 46451.776 & 0.554 & 0.869 & 35.5 & \\
\hline 1986 & 46451.904 & 0.637 & 0.952 & 34.5 & \\
\hline 1986 & 46452.016 & 0.710 & 0.025 & $\begin{array}{ll}30.9 & 0.3\end{array}$ & \\
\hline 1986 & 46452.712 & 0.162 & 0.477 & $\begin{array}{ll}37.8 & 0.7\end{array}$ & \\
\hline 1986 & 46452.821 & 0.233 & 0.548 & 32.3 & \\
\hline 1986 & 46453.766 & 0.847 & 0.162 & 36.9 & \\
\hline 1986 & 46453.937 & 0.958 & 0.273 & $34.7 \quad 0.5$ & \\
\hline 1986 & 46454.774 & 0.502 & 0.817 & $38.3 \quad 1.3$ & \\
\hline mean & & & & 34.51 .2 & \\
\hline 2005 & 53663.585 & 0.580 & 0.678 & $30.1 \quad 1.2$ & Rozhen \\
\hline 2006 & 53785.240 & 0.644 & 0.737 & $31.0 \quad 0.9$ & \\
\hline mean & & & & 30.5 & \\
\hline 2006 & 53818.313 & 0.889 & 0.981 & $38.2 \quad 1.5$ & Ondřejov \\
\hline 2006 & 54017.531 & 0.612 & 0.695 & $30.4 \quad 2.8$ & \\
\hline 2006 & 54017.577 & 0.642 & 0.725 & $34.8 \quad 1.7$ & \\
\hline 2006 & 54084.436 & 0.095 & 0.174 & $26.9 \quad 2.2$ & \\
\hline 2007 & 54115.439 & 0.244 & 0.322 & $30.6 \quad 1.5$ & \\
\hline 2007 & 54192.310 & 0.203 & 0.277 & $28.0 \quad 1.5$ & \\
\hline mean & & & & 31.51 .8 & \\
\hline
\end{tabular}

\subsection{Precession}

Shore \& Adelman (1976) proposed that $\mathrm{mCP}$ stars that are not members of binary systems could experience a free-body precession due to the distortion of the spherical shape of the star produced by the magnetic field.

The period change due to the precession is (see Appendix A) approximately

$$
\begin{aligned}
\frac{\dot{P}}{P} & =\Theta(\psi, \phi) \frac{4 \pi P}{P_{\mathrm{p}}^{2}} \\
& =3.4 \times 10^{-6} \text { year }^{-1} \Theta(\psi, \phi)\left(\frac{P}{1 \text { day }}\right)\left(\frac{P_{\mathrm{p}}}{100 \text { years }}\right)^{-2},
\end{aligned}
$$

where $P_{\mathrm{p}}$ is the period of precession, $P_{\mathrm{p}}=2 \pi / \omega_{\mathrm{p}}$, and $\Theta(\psi, \phi)$ is a function of the spherical coordinates $\psi$ and $\phi$ given by Eq. (A.6). From Fig. A.2 we conclude that explaining the period change of HD 37776 by precession gives too small a value for the angle $\psi<\pi / 3$ and for a minimum period of the precession $P_{\mathrm{p}}>120$ years, which can be inferred from the observed increase of the rotational period.

The total change of the phase during the time covered by the photometric and spectroscopic observations is about 0.48 (see Sect. 4.1). The maximum predicted change of phase due to precession for different parameters describing the precession is, according to Eq. (A.8), about 0.25 (see also Fig. A.3). The observed phase change therefore appears to be higher than what can be caused by the precession.

Precession will also cause a change in the inclination $i$ of the rotational axis of the star. Thus during the precession cycle the hemisphere visible at each rotation differs slightly from that visible during the previous one. As a result, one would then expect that the light curves would also change in shape and amplitude over several observing seasons. Such an effect has been documented on a time scale of only two years in the case of 56 Ari (Žižňovský et al. 2000). The detailed analysis carried out in Sect. 3.5 (see also Fig. 4) has shown that the shape of the light curves of HD 37776 has not changed during the last 31 years. This is further strong evidence against an explanation of the star's observed period increase in terms of precession.

\subsection{Evolutionary changes}

Changes in the internal structure of HD 37776 during its evolution could possibly provide another explanation of the observed rotational braking of object. However, stellar evolution models that include the effects of stellar rotation (Meynet \& Maeder 2000) do not show such a rapid change of stellar angular velocities during a star's main sequence evolution. Only at the very beginning of a star's main sequence stage, when the rotation profile adjusts rapidly, are the predicted changes in rotation comparable to those observed (Zahn 1992). However, it is questionable whether HD 37776 is in a very early phase of its main sequence evolution since it is considered a member of the Orion OB1b association, whose age is not less than $1 \mathrm{Myr}$ (Landstreet et al. 2007; Caballero 2007, and references therein). On the other hand even if the age of the Orion OB1b association is larger than $1 \mathrm{Myr}, \mathrm{HD} 37776$ itself may be one of younger objects that originated at the end of the region's star forming period.

\subsection{Continuous angular momentum loss}

Generally, photometric periods of $\mathrm{mCP}$ stars are constant (Mikulášek et al. 2007a) over timescales of decades, supporting the idea that they result from the rotation of rigid bodies. Consequently, in the following discussion, we shall assume that the period increase observed in HD 37776 results from rotational braking. Assuming rigid-body rotation, the period change, $\dot{P}$, due to the angular momentum loss per unit of time, $\dot{J}$, is

$\frac{\dot{P}}{P}=-\frac{\dot{J}}{J}=-\frac{\dot{J}}{\eta M R^{2} \Omega}$,

where $\eta \sim 0.05$ is a dimensionless constant appropriate for a star of a mass $M=9 M_{\odot}$ (Meynet \& Maeder 2006), $R$ is the stellar radius. The stellar angular momentum $J=\eta M R^{2} \Omega$, where $\Omega=2 \pi / P$.

One of the possible mechanisms that may cause the lengthening of the rotational period is the magnetic braking due to the momentum carried away by electromagnetic radiation emergent from the rotating magnetic field. Such a mechanism is thought to be responsible for the spindown of some pulsars. If the rotational braking is due to such electromagnetic torques, then the period change (assuming a dipolar magnetic field) is given by (e.g., Longair 1994)

$$
\begin{aligned}
\frac{\dot{P}}{P} \approx \frac{8 \pi^{2} R^{4} B_{0}^{2}}{3 \eta c^{3} M P^{2}}= & 4.9 \times 10^{-15} \text { year }^{-1} \frac{1}{\eta}\left(\frac{R}{1 R_{\odot}}\right)^{4}\left(\frac{B_{0}}{10^{4} \mathrm{G}}\right)^{2} \\
& \times\left(\frac{M}{1 M_{\odot}}\right)^{-1}\left(\frac{P}{1 \text { day }}\right)^{-2}
\end{aligned}
$$

where $B_{0}$ is the dipolar component of the surface magnetic field. The parameters appropriate for HD 37776 (assuming spectral type B2 V) are $M=8 M_{\odot}, R=4 R_{\odot}$ (Harmanec 1988) and $B_{0}=20 \mathrm{kG}$ (Khokhlova et al. 2000). Equation (25) then gives $\dot{P} / P=5.3 \times 10^{-12}$ year $^{-1}$, which is six orders of magnitude smaller than the observed value. 
Because magnetic braking alone seems to be too inefficient to slow the rotation rate of HD 37776, another process must be contributing to the observed period increase. The hot mCP stars are known to have stellar winds, so the momentum loss through a magnetically confined stellar wind may result in more efficient rotational braking (e.g., Mestel 2003). The loss of angular momentum in this case is $\dot{J}=\xi \dot{M} r_{\text {cor }}^{2} \Omega$, where $r_{\text {cor }}$ is the radius of the effective corotation, $\xi$ is a geometric factor and $\dot{M}$ is the mass-loss rate produced by the wind. Assuming that $r_{\mathrm{cor}}$ is given by the Alfvén radius and that the magnetic field intensity varies with radius as $B=B_{0}\left(\frac{R}{r}\right)^{n}$, the period change is

$$
\begin{aligned}
\frac{\dot{P}}{P} \approx & \frac{\xi \dot{M}}{\eta M}\left(\frac{B_{0}^{2} R^{2}}{\dot{M} v_{\infty}}\right)^{\frac{1}{n-1}}=10^{-13} \operatorname{year}^{-1}\left(7.8 \times 10^{8}\right)^{\frac{1}{n-1}} \frac{\xi}{\eta} \\
& \times\left(\frac{\dot{M}}{10^{-13} M_{\odot} \text { year }^{-1}}\right)\left(\frac{M}{1 M_{\odot}}\right)^{-1}\left(\frac{B_{0}}{10^{4} \mathrm{G}}\right)^{\frac{2}{n-1}}\left(\frac{R}{1 R_{\odot}}\right)^{\frac{2}{n-1}} \\
& \times\left(\frac{\dot{M}}{10^{-13} M_{\odot} \text { year }^{-1}}\right)^{-\frac{1}{n-1}}\left(\frac{v_{\infty}}{10^{3} \mathrm{~km} \mathrm{~s}^{-1}}\right)^{-\frac{1}{n-1}},
\end{aligned}
$$

where $v_{\infty}$ is the wind velocity at large radii (the terminal velocity). As the influence of quadrupolar and higher order components of the magnetic field is negligible at large radii we considered only dipolar component of the field: $n=3, \xi=0.1$, a typical mass-loss rate required to explain the He-rich peculiarity by diffusion theories (Michaud et al. 1987) $\dot{M} \sim 10^{-13} M_{\odot}$ year $^{-1}$, and a typical terminal velocity for $\mathrm{O}$ star winds of $v_{\infty}=1000 \mathrm{~km} \mathrm{~s}^{-1}$, we obtain $\dot{P} / P=6 \times 10^{-9}$ year $^{-1}$, still too low compared to the observed value, but much higher than for the case of pure magnetic braking.

On the other hand, the typical mass-loss rates predicted for B2 V stars are markedly higher, $\dot{M} \approx 10^{-9} M_{\odot}$ year $^{-1}$ (Krtička et al. 2006). Moreover, the magnetohydrodynamic calculations of winds confined by the magnetic field (ud-Doula \& Owocki 2002) predict a lower value of $n=\frac{11}{4}$ (ud-Doula, private communication, ud-Doula, Owocki \& Townsend, private communication). For these parameters we obtain $\dot{P} / P=2 \times 10^{-6}$ year $^{-1}$, which is in reasonable agreement with the observations.

If the outer layers of the star do not rotate as a rigid body then the rotational braking of the stellar surface caused by the angular momentum loss could be much faster. Internal differential rotation may not be unusual in main sequence stars as found by Yildiz (2004) for A-type components of binaries with well-determined stellar and binary parameters. The possibility of strong differential rotation as a result of external braking is also supported from a theoretical point of view since the typical decay time of differential rotation is comparable with the life time of A-type stars (Arlt et al. 2003, see also Zahn 1992).

The maximum age of HD 37776 derived by assuming a constant $\dot{P} / P$ (see Eq. (26)) throughout the main sequence lifetime of the star and critical rotation at the ZAMS is about 350000 years, which seems to be inconsistent with the age of the Orion OB1b association estimated by Landstreet et al. (2007) and Caballero (2007). However, the same argument given in Sect. 4.4 for a possible younger age of HD 37776 should also be taken into account. This discrepancy might also be explained by a lower mass-loss rate on the ZAMS than at the present time, by the accretion of external material that recently stopped, or by assuming that the braked surface layers have been getting shallower.

\subsection{Discrete angular momentum loss}

It is conceivable that the escape of material originating from a magnetically confined stellar wind does not occur continuously but may instead take place in discrete events. This is a scenario that has been suggested for CU Vir (Pyper et al. 1998; Trigilio et al. 2007). Such a process of gradual accumulation of material from the wind in the stellar magnetosphere, followed by the sudden breakout and escape of accumulated matter will result in an abrupt change of the rotation period each time it occurs. However, we again argue that there is little evidence for an abrupt change of the rotation period of HD 37776. The position and precision of the 1986 CFHT equivalent width measurements shown in Fig. 5 rule this out. Two linear regressions, one for the 1976-1982 data and the other for the 1991-2007 data, intersect at or close to 1988 when the maximum of the light curves should occur at phase $\lesssim 0.635$, far removed from the phase observed for the high $S / N$ CFHT data.

Nevertheless, it is interesting to estimate how much mass would have to be lost by HD 37776 to suddenly reduce its rotation period by the measured amount of $\Delta P=13.5 \pm 0.9 \mathrm{~s}$ (see Sect. 4.1). To do so, we will simply assume that all of the material with mass $\Delta M$ corotating in an axially symmetric ring around the star at a distance $r_{\text {cor }}$ suddenly escapes. If the star and its magnetosphere rotates as a rigid body, the required mass of the ring is given by

$$
\frac{\Delta M}{M}=\eta \frac{\Delta P}{P} \frac{R^{2}}{r_{\mathrm{cor}}^{2}}
$$

Since the corotation radius, $r_{\mathrm{cor}}$, in the rigidly rotating magnetospheric models of Townsend \& Owocki (2005) is on the order of a few stellar radii, the required mass of the corotating material is then on the order of $\Delta M \approx 10^{-7} M_{\odot}$. We argue that a stellar envelope containing such a quantity of matter, and its sudden loss, would not be photometrically or spectroscopically inconspicuous.

\subsection{Transient angular momentum loss}

If we accept the reality of the cubic term in the ephemeris of HD 37776, then this suggests that its gradual period increase will eventually cease. The observed period change of the star may then be a random episodic event. This might explain the discrepancy between the maximum age of HD 37776 derived from the observed period increase and the age of the Orion OB1b association. On the other hand, episodic mass-loss is not expected in the framework of classical radiation-driven wind theory (e.g., Krtička et al. 2006).

\subsection{Other stars}

Changes in rotation periods may not be rare among the mCP stars. Such changes have already been observationally confirmed in two stars.

A gradual increase in the rotation period at a rate of $\dot{P} / P \simeq$ $3 \times 10^{-7}$ year $^{-1}$ is exhibited by the rapidly rotating $(P \approx 0.7279)$ Si mCP star 56 Arietis (Musielok 1988; Adelman et al. 2001). For this star the period increase is also accompanied by apparent changes of its $U B V$ light curves (Adelman et al. 2001; Žižňovský et al. 2000). As discussed above, this is what is expected if the observed period changes are due to free body precession. However, in the case of HD 37776 such an explanation fails as the observed period changes are too large and they are 
not accompanied by corresponding changes of the shapes of its light curves.

Two abrupt period increases of one or two seconds have been detected in the very fast rotating $(P \approx 0 \mathrm{~d} 5207) \mathrm{Si} \mathrm{mCP}$ star CU Virginis (Pyper et al. 1998; Trigilio et al. 2007) in 1985 and 1998. CU Vir is also known to be a pulsar-like radio source (Leone et al. 1994; Kellett et al. 2007). The period jumps have been interpreted as a sudden spinning down of the star as a result of the violent emptying of the inner magnetosphere after the magnetic belt reaches a maximum density (Trigilio et al. 2007). The estimated mean value of $\dot{P} / P$ is $3.7 \times 10^{-6}$ year $^{-1}$, very close to the value $\simeq 4.0 \times 10^{-6}$ year $^{-1}$ we have derived for HD 37776 . In the case of CU Vir however, the nature of rotational braking appears different from the gradual spin-down of HD 37776. The latter object shows no evidence of discrete braking events and is not a source of radio emission (Linsky et al. 1992).

\section{Conclusions}

We have analysed all available observations of the magnetic, helium-strong star HD 37776 acquired since 1976. We find that the period of the light as well as spectroscopic variability has increased at the remarkable mean rate of $0.524 \pm 0.021 \mathrm{~s}$ per year, or $17.7 \pm 0.7$ s over the last 31 years. With $\dot{P} / P \simeq 4.0 \times 10^{-6}$ year $^{-1}$, the star has the largest relative increase of the rotation period known for any $\mathrm{mCP}$ star and, as a result, the largest measured braking of any star on the main sequence.

The period increase is interpreted in terms of a reduction in the rotation rate of HD 37776. The origin of this rotational braking is investigated from the point of view of i) a light-time effect on the rotation period as caused by an unseen, massive companion; ii) free-body precession of the rotational axis as a consequence of magnetic distortion of the spherical shape of the star; iii) evolutionary changes; iv) angular momentum loss due to pure magnetic braking or via a continuous or transient magnetically confined stellar wind. We have ruled out the light-time effect and free-body precession as possible causes. A forced precession, as may occur in multiple systems, is also excluded since radial velocity measurements of the star show no convincing evidence for variability at the expected level. The predicted angular momentum loss due to magnetic braking is found not to be effective enough to produce the observed rate of spin-down observed in HD 37776 if it is rotating as a rigid body. We found that the only realistic mechanism for rotational braking, at least for its surface layers, is angular momentum loss via its magnetically confined stellar wind. The possibility that the period increase was caused by a random, relatively short-lived (but not abrupt) transient event with a mass loss powerful enough to carry away a significant amount of the star's angular momentum can not be ruled out.

Assuming a cubic relation for the observed period change of HD 37776, Fig. 6 shows that the rate of the period increase is decreasing and should stop shortly after 2009. Further regular photometric, spectroscopic and magnetic monitoring of the star are obviously encouraged and will be very useful to extend the baseline of observations and confirm if this trend continues. Such new datasets will help to constrain further the possible physical causes of the unusual period behaviour, and in particular help decide if the observed period increase is a long-term phenomenon lasting for tens of thousand of years or a transient event with a duration of only a few tens of years.

It is interesting to note that three $\mathrm{mCP}$ stars have now been observed to show increases in rotation periods but in all three cases the suggested origin of the period change is different. The

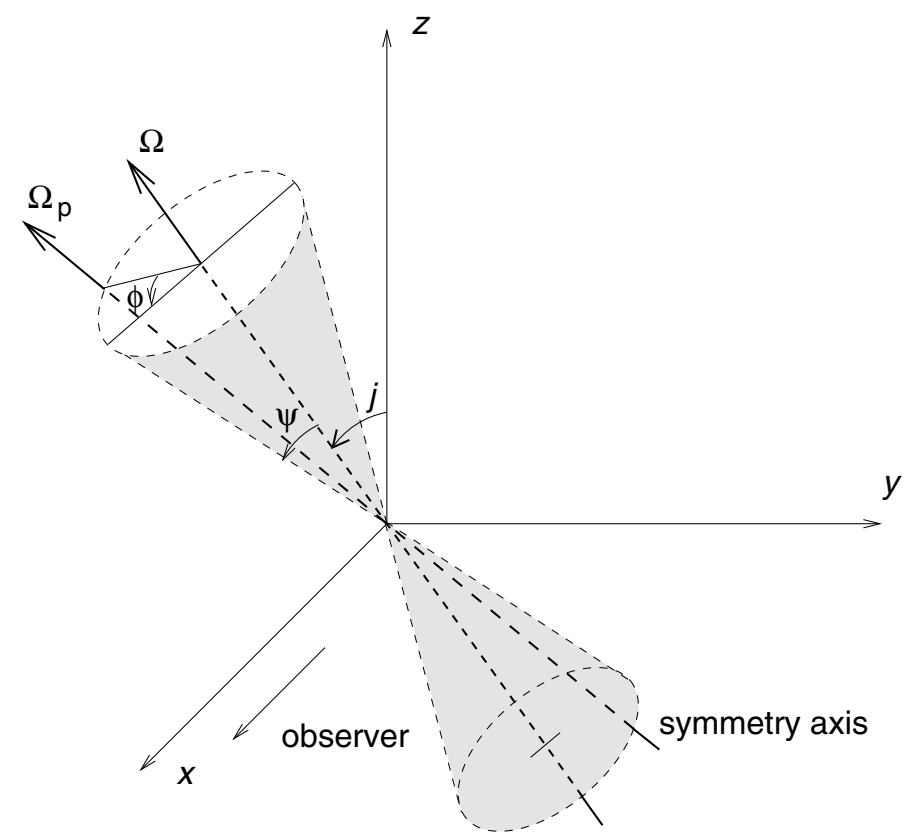

Fig. A.1. A geometrical illustration of the precession of the rotational axis of a star.

period increase observed in 56 Ari probably arises from free body precession (Adelman et al. 2001), abrupt changes in the period of CU Vir appear to be the result of sudden releases of trapped magnetospheric plasma (Trigilio et al. 2007) and, in this paper, we propose that magnetic braking, with the assistance of a magnetically confined wind, is the most likely cause of the steady, rapid increase in the rotation period of HD 37776 . High $S / N$ photometric and spectropolarimetric observations of these and other mCP stars and a thorough analysis of their eventual period changes are all likely to yield interesting new results.

Acknowledgements. The authors would like to thank Prof. John D. Landstreet for his valuable remarks and recommendations, as well as to Profs. P. Harmanec, S. Shore, Drs. D. Groote, S. Hubrig, J. Kubát, D. Kudryavtsev, P. North and M. Wolf for the discussion of the paper. This work was supported by grants GA ČR 205/06/0217, 205/06/0584, GA AV ČR KJB301630501, MVTS SR-ČR 10/15, MEB 080832, VEGA 2/6036/6 and Bulgarian NSF F-1403/2004. GWH acknowledges support from NASA, NSF, Tennessee State University, and the state of Tennessee through its Centers of Excellence program. This research has made use of NASA's Astrophysics Data System, the SIMBAD database, operated at CDS, Strasbourg, France and the On-line database of photometric observations of $m$ CP stars (Mikulášek et al. 2007a).

\section{Appendix A: Period change due to the precession}

Here we shall kinematically describe the change of the observed period due to the free precession of a distorted star assuming that the star behaves as a rigid body.

Let us first assume that the rotational axis $\boldsymbol{\Omega}$ is perpendicular to the direction to the observer, i.e. $j=0$. In the case of free precession, the symmetry axis (the star rotates around this axis with the frequency $\Omega_{p}$ ) moves on the surface of a cone with vertex angle $\psi$ (see Fig. A.1, e.g., Shaham 1986). The coordinates of the symmetry axis in a given instant of time are given by the angles $\psi$ and $\phi$, where $\phi$ is the angle between the plane given by $\boldsymbol{\Omega}$ and the direction to the observer and plane given by $\boldsymbol{\Omega}_{\mathrm{p}}$ 


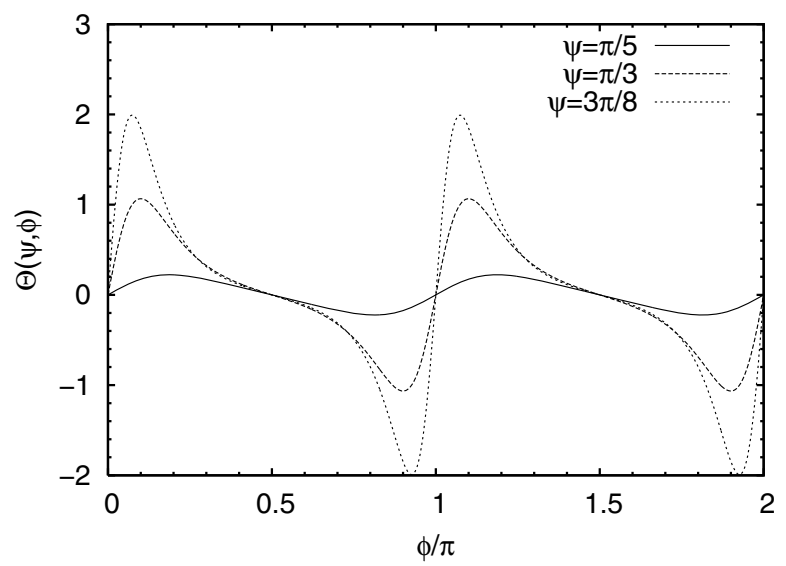

Fig. A.2. Plot of the function $\Theta(\psi, \phi)$ (see Eq. (A.6)) for different values of $\psi$.

and $\boldsymbol{\Omega}$. The coordinates of the point at the stellar equator with longitude $\varphi$ are given by

$x^{\prime}=\cos \phi \cos \psi \cos \varphi+\sin \phi \sin \varphi$,

$y^{\prime}=-\sin \phi \cos \psi \cos \varphi+\cos \phi \sin \varphi$,

$z^{\prime}=-\sin \psi \cos \varphi$.

If the rotational axis is tilted from the perpendicular direction by the angle $j$, then the coordinates of the stellar equator are given by

$$
\begin{aligned}
x= & \cos j(\cos \phi \cos \psi \cos \varphi+\sin \phi \sin \varphi) \\
& -\sin j \sin \psi \cos \varphi, \\
y= & -\sin \phi \cos \psi \cos \varphi+\cos \phi \sin \varphi, \\
z= & -\sin j(\cos \phi \cos \psi \cos \varphi+\sin \phi \sin \varphi) \\
& -\cos j \sin \psi \cos \varphi .
\end{aligned}
$$

The change of the phase due to the precession can be defined as the angle, $\Delta \phi$, by which we have to rotate the star back in such a way that the equatorial point with $\varphi=0$ is located in the plane $y=0$. From Eq. (A.2b)

$\sin \Delta \phi \cos \psi \cos \varphi=\cos \Delta \phi \sin \varphi$,

i.e.,

$\tan \Delta \phi=\frac{\tan \varphi}{\cos \psi}$.

The angle $\varphi$ changes linearly with time, $\varphi=\omega_{\mathrm{p}} t$, where $\omega_{\mathrm{p}}$ is the frequency of precession. The precession first modifies the rotational period $P^{\prime}=2 \pi / \Omega$ to

$P=P^{\prime}-\frac{\mathrm{d}}{\mathrm{d} t}\left(\frac{\Delta \phi}{2 \pi}\right) P^{\prime 2}=\left(1-\frac{\cos \psi}{1-\cos ^{2} \varphi \sin ^{2} \psi} \frac{\omega_{\mathrm{p}} P^{\prime}}{2 \pi}\right) P^{\prime}$.

Note that for $\omega_{\mathrm{p}} P^{\prime} \ll 1$ and $\psi<\pi / 2$ the period $P \approx P^{\prime}$. The period change is then (see Fig. A.2)

$\dot{P}=\frac{\sin \varphi \cos \varphi \sin ^{2} \psi \cos \psi}{\left(1-\cos ^{2} \varphi \sin ^{2} \psi\right)^{2}} \frac{\omega_{\mathrm{p}}^{2}}{\pi} P^{\prime 2} \equiv \Theta(\psi, \varphi) \frac{\omega_{\mathrm{p}}^{2}}{\pi} P^{\prime 2}$.

From Eq. (A.5) the period is, for $\omega_{\mathrm{p}}>0$, a minimum for $\varphi=0$ and equal to $P_{\min }=\left(1-\omega_{\mathrm{p}} P^{\prime} /(2 \pi \cos \psi)\right) P^{\prime}$.

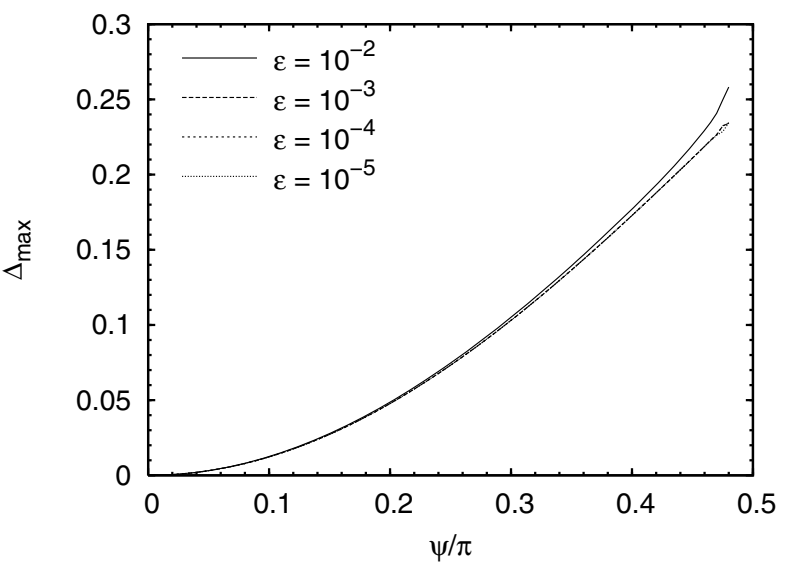

Fig. A.3. The maximum change of the rotational phase due to the precession $\Delta_{\max }$ (see Eq. (A.7)) as a function of angle $\psi$ for different ratios of the rotational period to $P^{\prime}$.

The period is at a maximum for $\varphi=\pi / 2$ and equal to $P_{\max }=\left(1-\cos \psi \omega_{\mathrm{p}} P^{\prime} /(2 \pi)\right) P^{\prime}$. The phase shift between these phases is equal to

$$
\begin{aligned}
\Delta_{\max } & =\int_{0}^{\frac{\pi}{2 \omega_{\mathrm{p}}}} \frac{\mathrm{d} t}{P}-\frac{\pi}{2 \omega_{\mathrm{p}} P_{\max }} \\
& =\int_{0}^{\frac{\pi}{2 \omega_{\mathrm{p}}}} \frac{\left(1-\cos ^{2} \varphi \sin ^{2} \psi\right) / P^{\prime} \mathrm{d} t}{1-\cos ^{2} \varphi \sin ^{2} \psi-\omega_{\mathrm{p}} P^{\prime} \cos \psi /(2 \pi)}-\frac{\pi}{2 \omega_{\mathrm{p}} P_{\max }} \\
& =\frac{\cos \psi \sin ^{2} \psi}{1-\varepsilon \cos \psi} \int_{0}^{\frac{\pi}{2}} \frac{\cos ^{2} \varphi}{1-\cos ^{2} \varphi \sin ^{2} \psi-\varepsilon \cos \psi} \frac{\mathrm{d} \varphi}{2 \pi}
\end{aligned}
$$

where $\varepsilon=P^{\prime} / P_{\mathrm{p}}$ and $P_{\mathrm{p}}=2 \pi / \omega_{\mathrm{p}}$. This equation can be analytically integrated but we give here only a plot (Fig. A.3) of $\Delta_{\max }$ as a function of $\psi$ for different periods of precession. For $\varepsilon \ll 1$ and $\psi<\pi / 2$ Eq. (A.7) does not depend on $\varepsilon$ and

$\Delta_{\max }=\frac{1}{4}(1-\cos \psi)$.

\section{References}

Abt, H. A. 1970, ApJS, 19, 387

Adelman, S. J. 1997, A\&AS, 125, 65

Adelman, S. J., \& Pyper, D. M. 1985, A\&AS, 62, 279

Adelman, S. J., Malanushenko, V., Ryabchikova, T. A., \& Savanov, I. 2001, A\&A, 375, 982

Arlt, R., Hollerbach, R., \& Rüdiger, G. 2003, A\&A, 401, 1087

Bartolini, C., Bonifazi, A., Fusi Pecci, F., et al. 1982, Ap\&SS, 83, 287

Bohlender, D. A. 1988, Ph.D. Thesis, Univ. of Western Ontario

Bohlender, D. A. 1994, in Pulsation, Rotation and Mass Loss in Early-Type Stars, ed. L. A. Balona, H. F. Henrichs, \& J. M. Le Contel (Dordrecht: Kluwer), IAU Symp., 162, 155

Borra, E. F., \& Landstreet, J. D. 1979, ApJ, 228, 809

Budaj, J., \& Komžík, R. 2001, http: //www .ta3.sk/budaj/software

Caballero, J. A. 2007, A\&A, 466, 917

Duflot, M., Figon, P., \& Meyssonnier, N. 1995, A\&AS, 114, 269

Eaton, J. A., Henry, G. W., \& Fekel, F. C. 2003, in The Future of Small Telescopes in the New Millennium, Volume II - The Telescopes We Use, ed. T. D. Oswalt (Dordrecht: Kluwer), 189

ESA 1997, in The Hipparcos and Tycho Catalogues, ESA SP-1200, Noordwijk Finkbeiner, D. P. 2003, ApJS, 146, 407

Groote, D., \& Kaufmann, J. P. 1983, A\&AS, 53, 91

Harmanec, P. 1988, Bull. Astron. Inst. Czechosl., 39, 329

Harmanec, P., Horn, J., \& Juza, K. 1994, A\&AS, 104, 121 
Henry, G. W. 1995a, in Robotic Telescopes: Current Capabilities, Present Developments, and Future Prospects for Automated Astronomy, ed. G. W. Henry, \& J. A. Eaton (San Francisco: ASP), ASP Conf. Ser., 79, 37

Henry, G. W. 1995b, in Robotic Telescopes: Current Capabilities, Present Developments, and Future Prospects for Automated Astronomy, ed. G. W. Henry, \& J. A. Eaton (San Francisco: ASP), ASP Conf. Ser., 79, 44

Hill, G. 1967, ApJS, 14, 263

Hunger, K. 1975, in Problems in Stellar Atmospheres and Envelopes (New York: Springer-Verlag), 57

Kellett, B. J., Graffagnino, V., Bingham, R., et al. 2007 [arXiv: astro-ph/0701214]

Khokhlova, V. L., Vasilchenko, D. V., Stepanov, V. V., \& Romanyuk, I. I. 2000, AstL, 26, 177

Knyazev, A. Yu., \& Shergin, V. S. 1995, SAO Technical Report, 239

Krtička, J., Kubát, J., \& Groote, D. 2006, A\&A, 460, 145

Krtička, J., Mikulášek, Z., Zverko, J., \& Žižňovský, J. 2007, A\&A, 470, 1089

Kudryavtsev, D. O. 2000, in Magnetic Fields of Chemically Peculiar and Related Stars, ed. Yu. V. Glagolevskij, \& I. I. Romanyuk (Spec. Astrophys. Obs.: Moscow), 84

Landstreet, J. D., Bagnulo, S., Andretta, V., et al. 2007, A\&A, 470, 685

Leone, F., Trigilio, C., \& Umana, G. 1994, A\&A, 263, 908

Linsky, L. L., Drake, S. A., \& Bastian, T. S. 1992, ApJ, 393, 341

Longair, S. M. 1994, High Energy Astrophysics (Cambridge: Cambridge University Press)

Lynds, C. R. 1959, ApJ, 130, 577

Mestel, L. 2003, Stellar Magnetism (Oxford: Oxford University Press)

Meynet, G., \& Maeder, A. 2000, A\&A, 361, 101

Meynet, G., \& Maeder, A. 2006, Stars with the B[e] Phenomenon, ed. M. Kraus, \& A. S. Miroschnichenko, ASP Conf. Ser., 355, 27

Michaud, G., Dupuis, J., Fontaine, G., et al. 1987, ApJ, 322, 302

Mikulášek, Z. 2007, Odessa Astron. Publ., in press [arXiv: 0711.4510]

Mikulášek, Z., Žižňovský, J., Zverko, J., et al. 2003, Contr. Astron. Obs. Skalnaté Pleso, 33, 29

Mikulášek, Z., Janík, J., Zverko, J., et al. 2007a, AN, 328, 10

Mikulášek, Z., Krtička, J., Zverko, J., et al. 2007b, in Active OB-Stars, ed. S. Štefl, S. P. Owocki, \& A. T. Okazaki (San Francisco: ASP Conf. Ser.), 466
Mikulášek, Z., Krtička, J., Zverko, J., et al. 2007c, in Physics of Magnetic Stars, Proceedings of the International Conference, held in the Special Astrophysical Observatory of the Russian AS, August 28-31, 2006, ed. I. I. Romanyuk, \& D. O. Kudryavtsev, 300

Musielok, B. 1998, IBVS, 3257

Neubauer, F. J. 1962, ApJ, 135, 748

Nissen, P. E. 1976, A\&A, 50, 343

Pedersen, H. 1979, A\&AS, 35, 313

Pedersen, H., \& Thomsen, B. 1977, A\&AS, 30, 11

Pojmański, G. 2002, Acta Astron., 52, 397

Pych, W. 2004, PASP, 116, 148

Pyper, D. M., Ryabchikova, T., Malanushenko, V., et al. 1998, A\&A, 339, 822 Romanyuk, I. I., Elkin, V. G., Kudryavtsev, D. O., Landstreet, J. D., \& Wade, G. A. 1998, Bull. of Spec. Astrophys. Obs., 45, 93

Shaham, J. 1986, ApJ, 310, 780

Shore, S. N. 1987, AJ, 94, 731

Shore, S. N., \& Adelman, S. J., 1976, ApJ, 209, 816

Shore, S. N., \& Brown, D. N. 1990, ApJ, 365, 665

Stibbs, D. W. N. 1950, MNRAS, 110, 395

Thompson, I. B., \& Landstreet, J. D. 1985, ApJ, 289, 9

Townsend, R. H. D., \& Owocki, S. P. 2005, MNRAS, 357, 251

Trigilio, C., Leto, P., Umana, G., Buemi, C. S., \& Leone, F., MNRAS, 384, 1437

Ud-Doula, A., \& Owocki, S. P. 2002, ApJ, 576, 413

van den Bergh, S. 1966, AJ, 71, 990

Vetö, B., Hempelmann, A., Schöneich, W., et al. 1991, AN, 312, 2

Warren, W. H., \& Hesser, J. E. 1978, ApJS, 36, 497

Wilson, R. E., \& Joy, A. 1950, ApJ, 111, 221

Yildiz, M. 2004, IAU Symp. No. 224, The A-Star Puzzle, ed. J. Zverko, J. Žižňovský, S. J. Adelman, \& W. W. Weiss (Cambridge: Cambridge University Press) 81

Zahn, J.-P. 1992, A\&A, 265, 115

Žižňovský, J., Schwartz, P., \& Zverko, J. 2000, IBVS, 4835

Zverko, J., Žižňovský, J., Mikulášek, Z., \& Iliev, I. Kh. 2007, Contr. Astron. Obs. Skalnaté Pleso, 37, 49 